gr-qc/9612020

\title{
Differential Forms and Wave Equations for General Relativity*
}

\author{
Stephen R. Lau ${ }^{\dagger}$ \\ Institut für Theoretische Physik, \\ Technische Universität Wien, \\ Wiedner Hauptstraße 8-10, \\ A-1040 Wien, Österreich
}

\begin{abstract}
Recently, Choquet-Bruhat and York and Abrahams, Anderson, ChoquetBruhat, and York (AACY) have cast the $3+1$ evolution equations of general relativity in gauge-covariant and causal "first-order symmetric hyperbolic form," thereby cleanly separating physical from gauge degrees of freedom in the Cauchy problem for general relativity. A key ingredient in their construction is a certain wave equation which governs the light-speed propagation of the extrinsic curvature tensor. Along a similar line, we construct a related wave equation which, as the key equation in a system, describes vacuum general relativity. Whereas the approach of AACY is based on tensor-index methods, the present formulation is written solely in the language of differential forms. Our approach starts with Sparling's tetrad-dependent differential forms, and our wave equation governs the propagation of Sparling's 2-form, which in the "time-gauge" is built linearly from the "extrinsic curvature 1-form." The tensor-index version of our wave equation describes the propagation of (what is essentially) the Arnowitt-Deser-Misner gravitational momentum.
\end{abstract}

Vienna, December 1996

\footnotetext{
*Preprint TUW-96-09 (revised). Research supported by the "Fonds zur Förderung der wissenschaftlichen Forschung" in Austria (Lise Meitner Fellowship M-00182-PHY and FWF Project 10.221-PHY).

†email address: lau@tph16.tuwien.ac.at
} 


\section{INTRODUCTION}

The $3+1$ formulation of general relativity views spacetime as the time history of the geometry associated with a spacelike hypersurface $\Sigma$. In the differential-forms (DF) version】 of $3+1$ relativity, the (co)triad 1-form $e^{\text {a }}$ and the "extrinsic curvature 1 -form" $K^{\text {a }}$ are taken as the basic geometrodynamical variables of interest. [1] In this description initial data sets $\left(e^{\mathrm{a}}, K^{\mathrm{a}}\right)$ are constrained by seven point-wise relations, DF versions of the familiar Hamiltonian, momentum, and rotation constraints; and the Arnowitt-Deser-Misner (ADM) [2,1] equations of motion for $e^{\mathrm{a}}$ and $K^{\mathrm{a}}$ govern the dynamic evolution of the system. These evolution equations preserve the constraints, and, moreover, maintain complete spatial covariance by means of an arbitrary shift vector $\beta^{j}$ and an arbitrary skew-symmetric rotation matrix $\phi_{\mathrm{ab}}$ (covariance under spatial coordinate transformations is not an issue in the DF language). However, as is well-known these equations do not manifest mathematically the propagation of physical degrees of freedom along the lightcone; they do not give rise to a physical wave equation. Therefore, in $3+1$ form relativity (as in its pure-index metric counterpart) gauge and physical variables remain intertwined.

Recently, Choquet-Bruhat and York [3] and Abrahams, Anderson, Choquet-Bruhat and York [4] (hereafter, we refer to both citations jointly as AACY) have cast the $3+1$ evolution equations for metric general relativity in gauge-covariant and causal "first-order symmetric hyperbolic form" (FOSH form), thereby cleanly separating physical from gauge degrees of freedom in the Cauchy problem for general relativity. A key ingredient in their construction is a certain wave equation which governs the light-speed propagation of the extrinsic curvature tensor $K_{i j}$. Along a similar line, we construct a related wave equation which, as the key equation in a system, describes general relativity. (Here we only consider vacuum, although we expect the inclusion of matter to be straightforward enough.) Whereas the approach of AACY is based on tensor methods, the present formulation is written solely in the language of differential forms. Our approach starts with Sparling's tetrad-dependent differential forms, [5, [6] and our wave equation governs the propagation of Sparling's 2-form, which in the "time-gauge" is built linearly from the extrinsic curvature 1-form. The tensorindex version of our wave equation describes the propagation of (what is essentially) the Arnowitt-Deser-Misner (ADM) gravitational momentum. As with AACY's "Box K" equation, to be proper our DF wave equation requires the implementation of a harmonic time slicing (or suitable generalization thereof) in order to handle the time re-parameterization invariance of Einstein's theory. In this paper we only derive the described wave equation. We do not discuss the possibility of constructing FOSH systems for Einstein's theory related to the one given by AACY. (We hope to return to this issue later.) Our goal is to understand the geometry of the AACY formalism in the DF language. Although there is no doubt in our mind that the original pure-index formulation of AACY is the superior wave-equation version of Einstein's theory, one might expect a DF construction to show distinct advantages in some theoretical contexts.

\footnotetext{
${ }^{1}$ Quite a number of reviews of "form relativity" exist. An excellent choice is the discussion found in Ref. [1] by Isenberg and Nester.
} 
AACY obtain their wave equation for $K_{i j}$ by (i) taking a further "time derivative" of the equation of motion for $K_{i j}$, and then (ii) subtracting from the resulting expression certain spatial covariant derivatives of the momentum constraint (which has $\beta^{j}$ as its corresponding Lagrange multiplier). Now, the standard DF version of $3+1$ relativity is, in essence, a triad theory, requiring the existence of three auxiliary phase-space constraints (packaged into one name, the "rotation constraint," with corresponding Lagrange multiplier $\left.\phi_{\text {ac }}\right)$. In our wave-equation construction, we proceed in a similar fashion to AACY. We also take the time derivative of an equation of motion and then subtract from the resulting expression certain spatial covariant derivatives of the momentum constraint. However, we find the need to also subtract from this time derivative certain spatial covariant derivatives of the rotation constraint. To us this seems to be natural generalization of the AACY method, necessary to handle the extra gauge freedom present in the triad theory. It is well-known that geometrically the rotation constraint is related to a certain piece of the spacetime torsion; and, therefore, in this paper we keep track of this very piece. Of course, this has consequences for the usual symmetries enjoyed by the Riemann, Ricci, and Einstein tensors (and DF versions thereof). However, we wish to stress here that we do not consider EinsteinCartan theory in this paper. "At the end of the day," we set all torsion equal to zero. We keep track of torsion in the beginning only as a rigorous way to handle the issue of the rotation constraint, which is always present in a triad version of Einstein's theory. [1.66.7]

The organization of this paper is as follows. In a preliminary section, $\S \mathrm{I}$, we fix our conventions and collect the geometric tools necessary for our central discussion. In particular, we quickly review the $3+1$ decomposition of spacetime differential forms and introduce a certain evolution operator $\hat{D}_{0}$, which may be regarded as a (triad) generalization of the evolution operator $\hat{\partial}_{0}$ central to the work of AACY. The reader may wish to simply peruse the preliminary section, and if necessary return to it later for more detailed study. § II deals with geometry of vacuum spacetimes as described by differential forms, and it lays the groundwork for our wave-equation formulation of general relativity. $\S$ III introduces Sparling's forms, using them to enact a DF version of the standard $3+1$ decomposition of spacetime geometry, while $\S$ IV combines the results of $\S$ II and III to write down a system of DF equations for general relativity, one equation in the system being the promised wave equation. We demonstrate the equivalence between our DF system and the original Einstein equations in $\S \mathrm{V}$. The concluding section provides some "instructions" for rewriting our results in tensor-index form. Finally, we have included as appendices a handful of technical derivations necessary for our central discussion.

\section{PRELIMINARIES}

\section{A. Notation and Conventions}

Consider a spacetime $\mathcal{M}$, equipped with Lorentz-signature metric $g_{\mu \nu}$, which is foliated

by a collection of spacelike 3-dimensional slices $\Sigma$. We use $\Sigma$ both to denote the foliation and to represent a generic slice. Since we are concerned only with local field equations in this work, we do not further specify the global properties of $\Sigma$. The timelike, future-pointing, 
unit, hypersurface normal of the $\Sigma$ foliation is $u^{\mu}$. Respectively, $h_{i j}$ and $K_{i j}$ denote the intrinsic metric and extrinsic curvature tensor associated with the $\Sigma$ foliation.

We shall use two types of spacetime frames in this work. One is the foliation-adapted "quasi-coordinate" frame $e_{\mu}$ (with coframe $e^{\mu}$ ) used by AACY

$$
\begin{aligned}
e^{0}=\mathrm{d} t & e_{0}=\partial / \partial t-\beta^{j} \partial / \partial x^{j} \\
e^{j}=\mathrm{d} x^{j}+\beta^{j} \mathrm{~d} t & e_{j}=\partial / \partial x^{j} .
\end{aligned}
$$

Greek letters $\{\mu, \nu, \lambda, \cdots\}$ run over $0,1,2,3$, but 0 is not $t$. Here $N$ and $\beta^{k}$ are respectively the standard lapse function and shift vector associated with the $\Sigma$ foliation. Lowercase Latin letters $\{i, j, k, \cdots\}$, taking the values $1,2,3$, represent the space legs of the quasi-coordinate frame and are also $\Sigma$ coordinate indices. We use $\partial_{0}$ to represent $e_{0}$ as a Pfaff derivative, 88 so for some scalar function $f$ we write $\partial_{0} f$ to mean $\dot{f}-\beta^{j} \partial f / \partial x^{j}$.

The second type of frame we consider is the foliation-adapted (or time-gauge) tetrad $e_{A}$ (with coframe $e^{A}$ ):

$$
\begin{array}{cc}
e^{\perp}=N e^{0} & e_{\perp}=N^{-1} e_{0} \\
e^{\mathrm{a}}=e^{\mathrm{a}}{ }_{j} e^{j} & e_{\mathrm{a}}=e_{\mathrm{a}}{ }^{j} e_{j} .
\end{array}
$$

Notice that $e_{\perp}^{\mu}$ is the $\Sigma$ normal $u^{\mu}$. Capital Latin letters $\{A, B, C, \cdots\}$ represent tetrad indices and run over $\perp, \hat{1}, \hat{2}, \hat{3}$, while block lowercase letters, typically from the first part of the alphabet, $\{\mathrm{a}, \mathrm{b}, \mathrm{c}, \cdots\}$ represent $\Sigma$ triad indices and run over $\hat{1}, \hat{2}, \hat{3}$. The set of $e_{\mathrm{a}}{ }^{j}$ comprise an orthonormal triad of vector fields on $\Sigma$. Orthonormality implies that $h_{i j} e_{\mathrm{a}}{ }^{i} e_{\mathrm{b}}{ }^{j}=\delta_{\mathrm{ab}}$, with $\delta_{\mathrm{ab}}=\delta^{\mathrm{ab}}=\operatorname{diag}(1,1,1)$. We can represent the $\Sigma$ metric as $h_{i j}=\delta_{\mathrm{ab}} e^{\mathrm{a}}{ }_{i} e^{\mathrm{b}}{ }_{j}$.

\section{B. 3+1 Splitting of Forms and the First Cartan Structure Equation}

We may split a general spacetime differential form $\Psi$ into pieces normal and tangential to the $\Sigma$ foliation,

$$
\Psi=\| \Psi+e^{\perp} \wedge \Psi_{\perp}
$$

where the $i_{\perp} \equiv i_{u}$ is the "inner derivative" with $u$, also known as the vector-form "hook" with $u$. The hook of a spatial form with the timelike $\Sigma$ normal $u=e_{\perp}$ vanishes. Both ${ }^{\|} \Psi$ and $\Psi_{\perp}$ are spatial forms. Let us take a special look at spacetime forms which happen to be spatial to start with. For the sake of definiteness only, consider a spatial 2 -form $\psi=\| \psi$. In the index notation and with the quasi-coordinate basis, the only non-vanishing components of $\psi_{\mu \nu}$ are $\psi_{i j}$, so that $\psi=\frac{1}{2} \psi_{i j} e^{i} \wedge e^{j}$. Note that, while $\psi$ is purely spatial, it is still a spacetime form; we have not pulled it back to a particular $\Sigma$ slice. This is an advantage gained by using the quasi-coordinate frame: all forms (in fact, all tensors) which are parallel to the $\Sigma$ foliation in spacetime may be represented with $\Sigma$ indices in the index notation. As another example, take $e^{\mathrm{a}}={ }^{\|} e^{\mathrm{a}}$, which has non-vanishing components $e^{\mathrm{a}}{ }_{j}$. 
Now suppose $\Psi$ is a 3 -form or less. We have the following decomposition of $\mathrm{d} \Psi$ with respect to the $\Sigma$ foliation: [1]

$$
\mathrm{d} \Psi=d^{\|} \Psi+N^{-1} e^{\perp} \wedge\left[\hat{\partial}_{0} \| \Psi-d\left(N \Psi_{\perp}\right)\right],
$$

where $\mathrm{d}$ is the $\mathcal{M}$ exterior derivative and $d$ is the $\Sigma$ exterior derivative. Also, our "timeevolution operator" is $\hat{\partial}_{0} \equiv £_{e_{0}}$, with $£$ representing $\mathcal{M}$ Lie differentiation.2 Note that $\hat{\partial}_{0}$ is not the Pfaff derivative $\partial_{0}=\partial / \partial t-\beta^{k} \partial / \partial x^{k}$, although it agrees with $\partial_{0}$ when acting on scalars. In the index notation, the action of $\hat{\partial}_{0}$ on a $\Sigma$ form, for example $\psi_{\lambda}=\psi_{i} e^{i}$, is given by $£_{e_{0}} \psi_{\lambda}=e_{\lambda}^{i}\left(\dot{\psi}_{i}-L_{\beta} \psi_{i}\right)$, with $L$ denoting intrinsic $\Sigma$ Lie differentiation. Thus, while working (in the index notation) with $\Sigma$ tensors carrying spatial indices $(i, j, k, \cdots)$, it is consistent to set $\hat{\partial}_{0}=\partial / \partial t-L_{\beta}$.

Applying the exterior derivative decomposition (1.4) twice to the formula $\mathrm{d}^{2} \psi=0$, where now $\psi=\| \psi$ is an arbitrary spatial form, one proves that the following commutator vanishes:

$$
\left[\hat{\partial}_{0}, d\right] \psi \equiv \hat{\partial}_{0} d \psi-d \hat{\partial}_{0} \psi=0
$$

In index notation (1.5) can be translated into the statement that $\hat{\partial}_{0}$ commutes with $\partial_{k}$ partial differentiation. However, for the Pfaff derivative $\left[\partial_{0}, \partial_{k}\right] \neq 0$.

Consider the first structure equation of Cartan,

$$
T^{A}=\mathcal{D} e^{A} \equiv \mathrm{d} e^{A}+\Gamma_{B}^{A} \wedge e^{B},
$$

where $T^{A}=\frac{1}{2} e_{\lambda}^{A} T_{\mu \nu}^{\lambda} e^{\mu} \wedge e^{\nu}$ is the torsion 2-form, the $\Gamma_{B}^{A}$ are the 1-forms specifying the spacetime connection with respect to the time-gauge tetrad (1.2), and $\mathcal{D}$ is the spacetime exterior covariant derivative. We shall assume that $T^{\perp}{ }_{i j}$ is the only piece of the spacetime torsion tensor which is not manifestly zero; hence we only need to consider the $T \equiv \| T^{\perp}$ piece of the torsion 2 -form.

The " projections of the $(\perp, a)$ values of $(1.6)$ tell us that

$$
\begin{aligned}
& 0=D e^{\mathrm{a}} \equiv d e^{\mathrm{a}}+\omega^{\mathrm{a}}{ }_{\mathrm{b}} \wedge e^{\mathrm{b}} \\
& T=K_{\mathrm{ab}} e^{\mathrm{a}} \wedge e^{\mathrm{b}},
\end{aligned}
$$

where $\omega_{\mathrm{b}}^{\mathrm{a}}=\| \Gamma_{\mathrm{b}}^{\mathrm{a}}$ are the $\Sigma$ triad connection 1-forms and $D$ is the $\Sigma$ exterior covariant derivative. The top equation says that the intrinsic connection on each slice $\Sigma$ is torsionfree. The second equation follows because (i) $e^{\perp} \wedge \mathrm{d} e^{\perp}=0$ and (ii) the triad components of the $\Sigma$ extrinsic curvature tensor are defined in the time-gauge by $K_{\mathrm{ab}} \equiv-\Gamma_{\mathrm{a} \perp \mathrm{b}}$. Therefore, setting $T$ equal to zero enforces the symmetry of $K_{i j}$. Taking the $i_{\perp}$ inner derivative of (1.6), one finds that

\footnotetext{
${ }^{2}$ It is precisely by choosing to take the Lie derivative along $e_{0}{ }^{\mu}$, that we may use spacetime $£$ in (1.4) in place of the "intrinsic $\Sigma$ Lie derivative generalized to take care of derivatives in directions off of the $\Sigma$ hypersurfaces" discussed in Ref. [1]. Had we chosen to take the Lie derivative along $u^{\mu}$, as is often done, then we would have needed to introduce such a generalized $\Sigma$ Lie derivative.
} 


$$
\begin{aligned}
& 0=\hat{\partial}_{0} e^{\mathrm{a}}+\Gamma^{\mathrm{a}}{ }_{\mathrm{b} 0} e^{\mathrm{b}}+N K^{\mathrm{a}}{ }_{\mathrm{b}} e^{\mathrm{b}} \\
& 0=-a_{\mathrm{b}}+\Gamma^{\perp}{ }_{\mathrm{b} \perp},
\end{aligned}
$$

with $a_{\mathrm{b}} \equiv e_{\mathrm{b}}[\log N]$. It follows that the $a^{\mathrm{b}}$ are the triad components of the acceleration of the $\Sigma$ foliation normal $u^{\mu}$. [1] Upon inspection of (1.8 a), we define a generalized version $\hat{D}_{0}$ of the evolution operator $\hat{\partial}_{0}$ by

$$
\hat{D}_{0} e^{\mathrm{a}} \equiv \hat{\partial}_{0} e^{\mathrm{a}}+\Gamma_{\mathrm{b} 0}^{\mathrm{a}} e^{\mathrm{b}}
$$

and will shortly extend its action to other forms and tensors.

\section{Triad-covariant Evolution Operator}

The advantage gained by using the simple evolution operator $\hat{\partial}_{0}$ is well-known. 12 Differentiation by $\hat{\partial}_{0}$ preserves the spatial character of any spatial form or spatial tensor. Moreover, $\hat{\partial}_{0}$ defines a good "time axis" as it is orthogonal to the spacelike $\Sigma$ foliation. Our generalization of $\hat{\partial}_{0}$, the degree-zero evolution operator $\hat{D}_{0}$ introduced in (1.9), is covariant with respect to triad or "internal" rotations. Now, in fact, the canonical formulation of triad gravity identifies the anti-commuting Lagrange parameter $\phi^{\mathrm{a}}{ }_{\mathrm{b}}$ associated with the rotation constraint as $-\Gamma^{\mathrm{a}}{ }_{\mathrm{b} 0}=-N \Gamma^{\mathrm{a}}{ }_{\mathrm{b} \perp}$. The connection coefficients $\Gamma^{\mathrm{b}}{ }_{\mathrm{a} 0}$ describe the rotation of the triad as it is parallel transported along the integral curves of $e_{0}{ }^{\mu}=N u^{\mu}$. We generalize the action of $\hat{D}_{0}$ to other triad tensor-valued forms in the obvious way. For example, consider a triad tensor-valued 0 -form $f_{\mathrm{a}} \mathrm{b}$. On such an object, the direct way of expressing the action of $\hat{D}_{0}$ would be

$$
\hat{D}_{0} f_{\mathrm{a}}^{\mathrm{b}}=D_{0} f_{\mathrm{a}}^{\mathrm{b}} \equiv \partial_{0} f_{\mathrm{a}}^{\mathrm{b}}-f_{\mathrm{c}}^{\mathrm{b}} \Gamma_{\mathrm{a} 0}^{\mathrm{c}}+f_{\mathrm{a}}^{\mathrm{c}} \Gamma^{\mathrm{b}}{ }_{\mathrm{c} 0}
$$

Notice that on 0 -forms $\hat{D}_{0}$ reduces to $D_{0}$. If we had taken $f_{\mathrm{a}}$ b as a tensor-valued 1 -form $f_{\mathrm{a}}^{\mathrm{b}}{ }_{k} e^{k}$, then we would have had to use the operator $\hat{\partial}_{0}$ in the first term on the right-most side of (1.10). In other words, the "hat" does not see triad indices, rather it sees whether or not the differentiated object is a form of degree higher than zero. Equation (1.8a) ensures that we have

$$
\hat{D}_{0} e^{\mathrm{a}}=-N K^{\mathrm{a}}
$$

with the extrinsic curvature appearing as a one-form $K^{\mathrm{a}}=K^{\mathrm{a}}{ }_{j} e^{j}$, as the evolution rule for the (co)triad.

Let us address some technical points concerning how to use the operator $\hat{D}_{0}$ in index notation. First, when working in index notation, assume the following evolution rules for the cotriad and triad:

$$
\begin{aligned}
\hat{D}_{0} e^{\mathrm{a}}{ }_{j} & \equiv \hat{\partial}_{0} e^{\mathrm{a}}{ }_{j}+e^{\mathrm{b}}{ }_{j} \Gamma^{\mathrm{a}}{ }_{\mathrm{b} 0}=-N K^{\mathrm{a}}{ }_{j} \\
\hat{D}_{0} e_{\mathrm{a}}{ }^{j} & \equiv \hat{\partial}_{0} e_{\mathrm{a}}{ }^{j}-e_{\mathrm{b}}{ }^{j} \Gamma^{\mathrm{b}}{ }_{\mathrm{a} 0}=N e_{\mathrm{b}}{ }^{j} K^{\mathrm{b}}{ }_{\mathrm{a}} .
\end{aligned}
$$


Second, assume that $\hat{D}_{0}$ obeys the Leibnitz rule and reduces to $\hat{\partial}_{0}$ when acting on tensors without triad indices. As an example of how to proceed in index notation, consider again our triad tensor-valued 0 -form, but assume that its triad components $f_{\mathrm{a}}{ }^{\mathrm{b}}=f_{i}{ }^{j} e_{\mathrm{a}}{ }^{i} e^{\mathrm{b}}{ }_{j}$ are obtained by "soldering" all of the free indices of a $\Sigma$ tensor $f_{i}{ }^{j}$. One can also obtain the action of $\hat{D}_{0}$ on $f_{\mathrm{a}}^{\mathrm{b}}$ by letting it act on $f_{i}{ }^{j} e_{\mathrm{a}}{ }^{i} e^{\mathrm{b}}{ }_{j}$ and then using the Leibnitz rule. The result is

$$
\hat{D}_{0} f_{\mathrm{a}}^{\mathrm{b}}=e_{\mathrm{a}}{ }^{i} e^{\mathrm{b}}{ }_{j} \hat{\partial}_{0} f_{i}{ }^{j}+N f_{\mathrm{c}}^{\mathrm{b}} K_{\mathrm{a}}^{\mathrm{c}}-N f_{\mathrm{a}}{ }^{\mathrm{c}} K^{\mathrm{b}}{ }_{\mathrm{c}} .
$$

Of course, (1.10) and (1.13) agree. By first writing $\delta_{\mathrm{ab}}=h_{i j} e_{\mathrm{a}}{ }^{i} e_{\mathrm{b}}{ }^{j}$ and $\epsilon_{\mathrm{abc}}=\epsilon_{i j k} e_{\mathrm{a}}{ }^{i} e_{\mathrm{b}}{ }^{j} e_{\mathrm{c}}{ }^{k}$, where $\epsilon_{i j k}$ is the $\Sigma$ permutation tensor fixed by $\epsilon_{123}=\sqrt{h}$, one can use the result $\hat{\partial}_{0} h_{i j}=$ $-2 N K_{(i j)}$ to show that $\hat{D}_{0}$ annihilates both $\delta_{\mathrm{ab}}$ and $\epsilon_{\mathrm{abc}}$. That $\hat{D}_{0}$ kills these particular triad tensors can also be shown with formulae like (1.10) along with the fact that the $\Gamma_{\text {ab0 }}$ are antisymmetric in their triad indices.

We have already seen that $\hat{\partial}_{0}$ and $d$ commute when acting on purely spatial forms. What about $\hat{D}_{0}$ and $D$ ? ( $D$ is the $\Sigma$ exterior covariant derivative.) Appendix B proves that for any triad-valued $\Sigma$ form $\psi^{\mathrm{a}}={ }^{\|} \psi^{\mathrm{a}}$, we have the following commutation rule:

$$
\left[\hat{D}_{0}, D\right] \psi^{\mathrm{a}} \equiv\left(\hat{D}_{0} D-D \hat{D}_{0}\right) \psi^{\mathrm{a}}=N\left(\Re_{\mathrm{b} \perp}^{\mathrm{a}}+a^{\mathrm{a}} K_{\mathrm{b}}-a_{\mathrm{b}} K^{\mathrm{a}}\right) \wedge \psi^{\mathrm{b}}
$$

where $\Re_{\mathrm{b} \perp}^{\mathrm{a}}=i_{\perp} \Re_{\mathrm{b}}^{\mathrm{a}}$ and the $\Re_{\mathrm{b}}^{\mathrm{a}}$ comprise the triad block of the matrix-valued curvature two-form of Cartan, $\Re_{B}^{A}=\frac{1}{2} \Re^{A}{ }_{B \mu \nu} e^{\mu} \wedge e^{\nu}$. Here the $\Re^{A}{ }_{B \mu \nu}=e^{A}{ }_{\alpha} e_{B}{ }^{\beta} \Re^{\alpha}{ }_{\beta \mu \nu}$ are the mixed components of the spacetime Riemann tensor.

\section{EINSTEIN GRAVITY IN TERMS OF DIFFERENTIAL FORMS}

Working within the Cartan framework of tensor-valued differential forms, in this section we collect a handful of standard results concerning the geometry of vacuum Einstein spacetimes. We carefully examine the role played by the residual torsion in our formalism, in particular deriving several identities which will be used later on to demonstrate that the residual torsion may safely removed.

\section{A. Einstein 3-form}

Consider the Einstein tensor $G_{\beta A} \equiv e_{A}{ }^{\alpha} G_{\beta \alpha}$ written with respect to a mixed basis. Here and it what follows we pay strict attention to index ordering. Since we are keeping track of certain components of the spacetime torsion, the Einstein tensor is not symmetric a priori in our formalism. We will work with the Einstein 3-form

$$
\mathrm{G}_{A} \equiv \frac{1}{6} G_{\kappa A} \epsilon^{\kappa}{ }_{\alpha \beta \lambda} e^{\alpha} \wedge e^{\beta} \wedge e^{\lambda} .
$$

Clearly, ${ }^{*} \mathrm{G}_{A}=G_{\beta A} e^{\beta}$, where $*$ denotes the Hodge-duality defined by the $\mathcal{M}$ metric $g_{\mu \nu}$. In (2.1) the $\mathcal{M}$ permutation tensor $\epsilon_{\lambda \mu \nu \sigma}$ is chosen so that $\epsilon_{0123}=\sqrt{-g}$. As described in the preliminary section, decompose the Einstein 3-form as 


$$
\mathrm{G}_{A}=\|_{A}+\mathrm{G}^{\perp} \wedge \mathrm{G}_{A \perp}
$$

Letting $\star$ denote the Hodge-duality defined by the $\Sigma$ metric $h_{i j}$, we have the relations ${ }^{\star} \mathrm{G}_{A \perp}=-G_{j A} e^{j}$ and ${ }^{\star} \mathrm{G}_{A}=-G_{\perp A}$. When working with the $\star$ duality in index notation, the $\Sigma$ permutation tensor $\epsilon_{i j k}$ is the appropriate one to use.

Let us write down DF equations which capture the antisymmetric pieces of the Einstein tensor. These will be related to the residual torsion $T_{i j}$ in the next subsection. First, one immediately finds that

$$
G_{i j} e^{i} \wedge e^{j}=e^{\mathrm{a}} \wedge^{\star} \mathrm{G}_{\mathrm{a} \perp}
$$

Now turn to the antisymmetry in the cross components $G_{\perp j}-G_{j \perp}$. We have already seen that ${ }^{\star} \mathrm{G}_{\mathrm{a}}=-G_{\perp \mathrm{a}}$. To get the other cross term look at $\mathrm{G}_{\perp \perp}$, which obeys ${ }^{\star} \mathrm{G}_{\perp \perp}=-G_{j \perp} e^{j}$. Therefore, the 1-form expression

$$
\left(G_{\perp j}-G_{j \perp}\right) e^{j}=-\left({ }^{\star} \|_{\mathrm{a}}\right) e^{\mathrm{a}}+{ }^{\star} \mathrm{G}_{\perp \perp}
$$

captures the cross-term antisymmetry.

\section{B. Bianchi Identity for the Torsion 2-form}

In this subsection we shall relate the residual torsion $T$ to the antisymmetric pieces of the Einstein tensor. To do this, we apply the form decompositions (1.3,1.4) to the Bianchi identity for the torsion,

$$
\mathcal{D} T^{A} \equiv \mathrm{d} T^{A}+\Gamma_{B}^{A} \wedge T^{B}=\Re_{B}^{A} \wedge e^{B},
$$

subject to the assumption that both $T^{\mathrm{a}}=0$ and $i_{\perp} T^{\perp}=0$.

The $\perp$ value of $(2.5)$ is then

$$
\mathrm{d} T=\Re^{\perp} \wedge e^{\mathrm{a}}
$$

The ${ }^{\|}$projection of (2.6) leads to an equation for the $\Sigma$ expression $d T$ which is of no concern to us here. However, taking the $i_{\perp}$ inner derivative of (2.6) and using the form decompositions (1.3, 1.4), we obtain the following identity:

$$
N^{-1} \hat{\partial}_{0} T=\Re^{\perp}{ }_{\mathrm{a} \perp \mathrm{b}} e^{\mathrm{b}} \wedge e^{\mathrm{a}} .
$$

In order to re-express (2.7) in terms of the Einstein 3 -form, first use the relations $\Re^{\perp}{ }_{\mathrm{a} \perp \mathrm{b}}=$ $e_{\mathrm{a}}{ }^{i} e_{\mathrm{b}} \Re_{i j}-\Re_{\text {acb }}^{\mathrm{c}}$ and $\Re_{[i j]}=G_{[i j]}$ to rewrite the preceding identity as

$$
G_{i j} e^{i} \wedge e^{j}=-N^{-1} \hat{\partial}_{0} T+\Re_{\mathrm{acb}}^{\mathrm{c}} e^{\mathrm{a}} \wedge e^{\mathrm{b}}
$$

\footnotetext{
${ }^{3}$ In these formulae $\Re_{i j}$ represents the spatial components of the spacetime Ricci tensor.
} 
Next, plug the Gauss-Codazzi-Mainardi (GCM) equation (A1a) into (2.8), use the fact that the $\Sigma$ Ricci tensor $R_{i j}$ is symmetric (since we have spatial torsion $T^{i}{ }_{j k}=0$ ), and appeal to (2.3). These steps lead to a key identity

$$
\hat{\partial}_{0} T=N H T+N K_{\mathrm{a}} \wedge^{\star}\left(e^{\mathrm{a}} \wedge^{\star} T\right)-N e^{\mathrm{a}} \wedge^{\star} \mathrm{G}_{\mathrm{a} \perp},
$$

which gives the "time derivative" $\hat{\partial}_{0} T$ of the our residual torsion. This important equation establishes that, given the vanishing of the torsion $\left.T\right|_{\Sigma}$ pulled back to some initial Cauchy surface, no torsion can develop for vacuum spacetimes characterized by a vanishing Einstein 3 -form.

We now turn to the triad values of the Cartan-Bianchi identity (2.5) for the torsion, which for our restricted torsion take the following form:

$$
\Gamma_{\perp}^{\mathrm{a}} \wedge T=\Re_{B}^{\mathrm{a}} \wedge e^{\mathrm{B}} .
$$

Using the GCM equation (A1 a) for the $3+1$ splitting of the triad components of the spacetime Riemann tensor, one finds that the " projection (2.10) is an identity which is trivially satisfied. However, taking the $i_{\perp}$ inner derivative of (2.10), one obtains

$$
a^{\mathrm{a}} T=\Re_{\mathrm{b} \perp}^{\mathrm{a}} \wedge e^{\mathrm{b}}+\Re_{\perp}^{\mathrm{a}},
$$

which after a few more manipulations yields

$$
\left.{ }^{\star} \| \mathrm{G}^{\mathrm{a}}\right) e_{\mathrm{a}}^{\star}-\mathrm{G}_{\perp \perp}=a \wedge^{\star} T .
$$

Here and in what follows we make extensive use of the following the common short-hands:

$$
\begin{aligned}
e_{\mathrm{ab}}^{\star} & \equiv \epsilon_{\mathrm{abc}} e^{\mathrm{c}} \\
e_{\mathrm{a}}^{\star} & \equiv \frac{1}{2} \epsilon_{\mathrm{abc}} e^{\mathrm{b}} \wedge e^{\mathrm{c}} .
\end{aligned}
$$

One should understand that ${ }^{\star} \mathrm{G}^{\mathrm{a}}$ is a 0 -form. Looking back at (2.3), we see that this identity relates the torsion 2-form to the cross-term antisymmetry of the Einstein tensor. In index notation, the equation

$$
G_{i \perp}-G_{\perp i}=T_{i j} a^{j}
$$

captures this antisymmetry.

\section{SPARLING FORMS AND THE $3+1$ EINSTEIN EQUATIONS}

Sparling's differential forms have played a central role in several recent developments in general relativity, [13] and the tetrad versions [5, 6] of these forms also play a principal role in our formalism. We begin this section by introducing the tetrad Sparling forms and by reviewing their remarkable properties. In particular, we recall that the Sparling forms provide a handy expression for the Einstein 3-form. Meshing quite nicely with our decomposition (11.4) of exact spacetime forms, this handy expression leads to a $3+1$ decomposition of the Einstein 3-form which is particularly useful in our construction of a nonlinear wave equation for general relativity. We consider the special case when the (tetrad-dependent) Sparling forms are determined by a time-gauge tetrad (1.2). Although the results of this section are standard ones, it would appear that we perform the $3+1$ decomposition in a novel way. 


\section{A. Basic Properties and Time-gauge Expressions}

We start with the Sparling relation, [5].6] which expresses the Einstein 3-form

$$
\mathrm{G}_{A}=\mathrm{d} \sigma_{A}-\tau_{A}-\frac{1}{2} \epsilon_{A B C D} T^{B} \wedge \Gamma^{C D}
$$

in terms of the torsion 2-form, the tetrad connection coefficients, and the tetrad-dependent Sparling 2-forms and 3-forms,

$$
\begin{aligned}
\sigma_{A} & \equiv-\frac{1}{2} \Gamma^{B C} \wedge e_{A B C}^{*} \\
\tau_{A} & \equiv \frac{1}{2}\left(\Gamma_{A}{ }^{B} \wedge \Gamma^{C D} \wedge e_{B C D}^{*}-\Gamma_{D}^{B} \wedge \Gamma^{D C} \wedge e_{A B C}^{*}\right) .
\end{aligned}
$$

Here $e_{A B C}^{*} \equiv \epsilon_{A B C D} e^{D}$. Notice that, due to the way in which the tetrad connection coefficients appear in the definitions above, the Sparling forms do not behave homogeneously under tetrad transformations. However, while individually neither $\mathrm{d} \sigma_{A}, \tau_{A}$, nor the final torsional term in the Sparling relation (3.1) behave homogeneously, the sum of terms on the right-hand side of (3.1) must behave homogeneously under tetrad transformations, since this sum is the Einstein 3 -form.

We shall have need to consider only the Sparling forms $\left\{\sigma_{\perp}, \sigma_{\mathrm{a}}, \tau_{\perp}, \tau_{\mathrm{a}}\right\}$ determined by a time-gauge tetrad (1.2). Consider first the $\|$ and ${ }^{\perp}$ projections of the 2 -forms $\left\{\sigma_{\perp}, \sigma_{\mathrm{a}}\right\}$. One quickly finds the following list:

$$
\begin{aligned}
{ }_{\sigma_{\mathrm{a}}} & =e_{\mathrm{ab}}^{\star} \wedge K^{\mathrm{b}} \\
\sigma_{\mathrm{a} \perp} & =\omega_{\mathrm{a}}+a^{\mathrm{b}} e_{\mathrm{ab}}^{\star} \\
{ }^{\sigma_{\perp}} & =\omega_{\mathrm{a}} \wedge e^{\mathrm{a}} \\
\sigma_{\perp \perp} & =N^{-1} \Gamma_{\mathrm{a} 0} e^{\mathrm{a}}
\end{aligned}
$$

where for notational convenience we define $\omega_{\mathrm{a}} \equiv-\frac{1}{2} \epsilon_{\mathrm{abc}} \omega^{\mathrm{bc}}$. We make the same definitions for $\Gamma_{\mathrm{a} 0}$ and the triad-valued curvature two-form $R_{\mathrm{a}}$ below in $(3.5 \mathrm{c})$. Notice that the forms ${ }^{~} \sigma_{\mathrm{a}}$ and $\sigma_{\mathrm{a} \perp}-\omega_{\mathrm{a}}$, behave homogeneously under triad transformations, and hence that it makes sense to consider their exterior covariant derivatives, $D^{\|} \sigma_{\mathrm{a}}$ and $D\left(\sigma_{\mathrm{a} \perp}-\omega_{\mathrm{a}}\right)$. Evidently, the expressions for $\sigma_{\perp \perp}$ and $\|_{\perp}$ behave inhomogeneously under rotations of the triad.

The task of finding the explicit time-gauge expressions for the set $\left\{{ }^{{ }} \tau_{\mathrm{a}}, \tau_{\mathrm{a} \perp},{ }^{{ }} \tau_{\perp}, \tau_{\perp \perp}\right\}$ is somewhat tedious, and to get the expressions in the list below, one must make extensive use of $\epsilon_{\mathrm{abc}}$ permutation-symbol gymnastics. We find the following set:

$$
\begin{aligned}
{ }_{\tau_{\mathrm{a}}}= & -\omega^{\mathrm{b}}{ }_{\mathrm{a}} \wedge K^{\mathrm{c}} \wedge e_{\mathrm{bc}}^{\star}+\frac{1}{2} \epsilon_{\mathrm{abc}} \omega^{\mathrm{bc}} \wedge T \\
{ }_{\tau_{\perp}}= & \frac{1}{2} K^{\mathrm{a}} \wedge K^{\mathrm{b}} \wedge e_{\mathrm{ab}}^{\star}-\frac{1}{2} \omega^{\mathrm{a}}{ }_{\mathrm{d}} \wedge \omega^{\mathrm{db}} \wedge e_{\mathrm{ab}}^{\star} \\
\tau_{\mathrm{a} \perp}= & -N^{-1} \Gamma^{\mathrm{b}}{ }_{\mathrm{a} 0} K^{\mathrm{c}} \wedge e_{\mathrm{bc}}^{\star}+R_{\mathrm{a}}-d \omega_{\mathrm{a}}-a \wedge \omega_{\mathrm{a}} \\
& +a^{\mathrm{b}} \omega^{\mathrm{c}}{ }_{\mathrm{a}} \wedge e_{\mathrm{bc}}^{\star}+\frac{1}{2} \epsilon_{\mathrm{abc}} K^{\mathrm{b}} \wedge K^{\mathrm{c}}-N^{-1} \Gamma_{\mathrm{a} 0} T \\
\tau_{\perp \perp}= & -a^{\mathrm{a}} K^{\mathrm{b}} \wedge e_{\mathrm{ab}}^{\star}-\frac{1}{2} \epsilon_{\mathrm{abc}} K^{\mathrm{a}} \wedge \omega^{\mathrm{bc}}-N^{-1} \Gamma^{\mathrm{a}}{ }_{\mathrm{d} 0} \omega^{\mathrm{db}} \wedge e_{\mathrm{ab}}^{\star}
\end{aligned}
$$

where in $(3.5 \mathrm{c})$ one finds the $\Sigma$ triad-valued curvature 2 -form $R_{\mathrm{a}}=d \omega_{\mathrm{a}}+\frac{1}{2} \epsilon_{\mathrm{abc}} \omega^{\mathrm{b}} \wedge \omega^{\mathrm{c}}$. 
We shall be dealing exclusively with the projected 2 -form ${ }^{\|} \sigma_{\mathrm{a}}$. Therefore, to avoid undue notational clutter, let us define the $\Sigma$ form

$$
\pi_{\mathrm{a}} \equiv-\frac{1}{2} \|_{\mathrm{a}}=\frac{1}{2} K^{\mathrm{b}} \wedge e_{\mathrm{ab}}^{\star}
$$

(the factor of $-\frac{1}{2}$ has been chosen for later convenience) and collect a few results concerning it. First, with the definition (2.13b), the evolution rule (1.11) for the cotriad shows that

$$
\hat{D}_{0} e_{\mathrm{a}}^{\star}=-2 N \pi_{\mathrm{a}} .
$$

Moreover, defining

$$
\pi^{\mathrm{ab}} \equiv e^{\mathrm{a}} \wedge \pi^{\mathrm{b}}=\frac{1}{2}\left(H \delta^{\mathrm{ab}}-K^{\mathrm{ab}}\right) e^{\star}
$$

we take the rotation-constraint 3-form to be

$$
J^{\mathrm{ab}}=-4 \pi^{[\mathrm{ab}]} .
$$

In fact, $J^{\mathrm{ab}}=\delta^{\mathrm{ac}} \delta^{\mathrm{bd}} T_{\mathrm{cd}} e^{\star}$, where the $T_{\mathrm{cd}}$ are the triad components of the residual torsion 2-form. Alternatively, the construction

$$
T=2 e^{\mathrm{a}} \wedge^{\star} \pi_{\mathrm{a}}
$$

provides a direct way of expressing the torsion 2 -form.

We wish to point out that 2 -form $\pi_{\mathrm{a}}$ is essentially the momentum conjugate to the cotriad in the Hamiltonian formulation of triad gravity. [6] Indeed, (switching to index notation) the $\Sigma$ Hodge dual of $\pi_{\text {aij }}$ is

$$
{ }^{\star} \pi_{\mathrm{a}}{ }^{k}=8 \pi h^{-1 / 2} e_{\mathrm{a} i} \pi_{A D M}^{k i},
$$

where $\pi_{A D M}^{i j}=(16 \pi)^{-1} \sqrt{h}\left(K h^{i j}-K^{i j}\right)$ is the standard ADM gravitational momentum. 2] Therefore, were we considering the standard Hamiltonian triad formalism, we would have the following as a canonical pair:

$$
\left\{(8 \pi)^{-1} \pi_{\mathrm{a} i j}, \tilde{e}^{\mathrm{a} i j}\right\}
$$

where $\tilde{e}^{\mathrm{aij}} \equiv \sqrt{h} \epsilon^{k i j} e^{\mathrm{a}}{ }_{k}$. The standard rotation constraint which arises in Hamiltonian triad gravity (also known as the Gauss constraint in the Ashtekar formulation [7]) is nothing but our $J^{\text {ab }}$ (stripped of a $d^{3} x$ ). The vanishing of the rotation constraint is equivalent to the vanishing of our residual torsion 2 -form $T$.

\footnotetext{
${ }^{4}$ Note that this is a special definition, and that $\pi^{\mathrm{ab}}$ is not obtained from $\pi^{\mathrm{b}}$ via inner differentiation, i. e. $\pi_{\mathrm{ab}} \not \equiv i_{\mathrm{b}} \pi_{\mathrm{a}}$.
} 


\section{B. 3+1 Decomposition of the Einstein 3-form}

Using the form decompositions (1.3) and (1.4) and recalling that the only non-zero piece of the torsion 2-form is $T=\| T^{\perp}$, we immediately write the Sparling relation (3.1) as the following $\perp$ and $\|$ projections:

$$
\begin{aligned}
\mathrm{G}_{A \perp} & =N^{-1}\left[\hat{\partial}_{0} \|_{A}-d\left(N \sigma_{A \perp}\right)\right]-\tau_{A \perp}-\eta_{A}^{\mathrm{b}} N^{-1} \Gamma_{\mathrm{b} 0} T \\
{ }^{\|} \mathrm{G}_{A} & =d{ }^{\|} \sigma_{A}-{ }^{\|} \tau_{A}-\eta_{A}^{\mathrm{b}} T \wedge \omega_{\mathrm{b}} .
\end{aligned}
$$

Now, in fact, we know that as a whole these pieces must be triad-gauge covariant. Indeed, insertion of the explicit expressions (3.4) and (3.5) into (3.13) yields the following nice formulae:

$$
\begin{aligned}
{ }^{\| \mathrm{G}_{\mathrm{a}}=} & -2 D \pi_{\mathrm{a}} \\
{ }^{\| \mathrm{G}_{\perp}=} & 2^{\star} \pi_{\mathrm{ab}} \pi^{\mathrm{ba}}-H \pi^{\mathrm{b}}{ }_{\mathrm{b}}+e^{\mathrm{a}} \wedge R_{\mathrm{a}} \\
\mathrm{G}_{\mathrm{a} \perp}= & -2 N^{-1} \hat{D}_{0} \pi_{\mathrm{a}}+N^{-1} e_{\mathrm{ab}}^{\star} \wedge D\left(N a^{\mathrm{b}}\right)-R_{\mathrm{a}} \\
& +2^{\star} \pi_{\mathrm{bc}}{ }^{\star} \pi^{\mathrm{cb}} e_{\mathrm{a}}^{\star}-H^{2} e_{\mathrm{a}}^{\star}-4^{\star} \pi_{\mathrm{ba}} \pi^{\mathrm{b}}+2 H \pi_{\mathrm{a}},
\end{aligned}
$$

where $H={ }^{\star} \pi^{\mathrm{a}}{ }_{\mathrm{a}}$ and $\pi^{\mathrm{a}}{ }_{\mathrm{a}}=H e^{\star}$. Notice that $(3.14 \mathrm{a})$ and $3.14 \mathrm{~b}$ ), respectively, are 3-form versions of the standard momentum and Hamiltonian constraints. We could effortlessly obtain the $3+1$ expression for $\mathrm{G}_{\perp \perp}$ with (3.14a) and our previously derived result (2.12).

\section{WAVE EQUATION FOR GENERAL RELATIVITY}

Let us quickly take stock of the situation thus far. As a statement of the standard $3+1$ version of Einstein's theory in vacuo, we take Eq. (3.7) (in practice, the equation of definition for $\pi_{\mathrm{a}}$ ), the vanishing of Eq. (3.14c) (the equation of motion for $\pi_{\mathrm{a}}$ ), and the vanishing of Eqs. (3.9), (3.14a), and (3.14b) (rotation, momentum, and Hamiltonian constraints). After making a short digression in $\S$ IV.A in order to discuss the Laplacian and wave operators we shall use in our construction, we introduce in $\S$ IV.B a certain triad-valued 2-form $\Upsilon_{\mathrm{a}}$ whose vanishing will, in effect, replace the evolution equation for $\pi_{\mathrm{a}}$. We then perform a $3+1$ decomposition on the 2 -form $\Upsilon_{a}$. This decomposition yields a non-linear wave equation for $\pi_{\mathrm{a}}$ (assuming a harmonic time-slicing) which serves as the key equation in our system of DF equations, written down in $\S$ IV.C, which describes vacuum general relativity.

\footnotetext{
${ }^{5}$ In [1] Isenberg and Nester expressed the gravitational constraints in essentially this way. The $\pi^{a}$ defined in a footnote on page 60 of that reference is, apart from an overall factor of -4 , the $\pi_{\mathrm{a}}$ that we use here. Also, their $\pi^{a b}$ differs from our $\pi^{\mathrm{ab}}$ by a factor of -2 .
} 


\section{A. Digression: Laplacian and Wave Operators on $\Sigma$ Triad-valued 2-forms}

Define a triad-covariant Laplacian $\Delta$ whose action on triad-valued $\Sigma 2$-forms $\psi_{\mathrm{a}}={ } \psi_{\mathrm{a}}$ is

$$
\Delta \psi_{\mathrm{a}} \equiv\left({ }^{\star} D^{\star} D-D^{\star} D^{\star}\right) \psi_{\mathrm{a}} .
$$

Clearly this operator reduces to the standard DF Laplacian when acting on ordinary 2-forms. Our chief interest is the action of the triad-covariant Laplacian $\Delta$ on the 2 -form $\pi_{\mathrm{a}}$. A short calculation shows that

$$
\Delta \pi_{\mathrm{a}}=e_{\mathrm{a}}{ }^{j} e^{\mathrm{b} i}\left(\bar{\nabla}_{m} \bar{\nabla}^{m} \pi_{i j}-\pi_{m j} R_{i}^{m}-\pi^{m p} R_{p j i m}\right) e_{\mathrm{b}}^{\star},
$$

where $\pi_{i j}=\frac{1}{2}\left(H h_{i j}-K_{i j}\right)$ and, in accord with the notation of AACY, we use $\bar{\nabla}_{i}$ to denote the torsion-free covariant derivative operator compatible with $h_{i j}$. Note that $\pi^{i j}$ differs from $\pi_{A D M}^{i j}$ by the density factor $(8 \pi)^{-1} \sqrt{h}$. Also, $R_{i j}$ is the $\Sigma$ Ricci tensor and $R_{i j k l}$ is the $\Sigma$ Riemann tensor. Clearly, even upon passage to the tensor-index formalism $\left({ }^{\star} D^{\star} D-D^{\star} D^{\star}\right)$ differs from the tensor $\bar{\nabla}^{k} \bar{\nabla}_{k}$ used in AACY. However, provided that equivalence with the Einstein equations has been shown, the extra curvature terms buried in the form Laplacian can be eliminated in favor of the variables of interest via a definite prescription. [3, 3, 9] The ability to eliminate $\Sigma$ curvature terms has proven to be crucial in AACY's construction of a (gauge-covariant and causal) FOsH system for Einstein's theory.

Our wave operator is

$$
\hat{\square}_{\pi_{\mathrm{a}}} \equiv\left[-N^{-2}\left(\hat{D}_{0}\right)^{2}+\Delta\right] \pi_{\mathrm{a}}
$$

Writing $\hat{\square} \pi_{\mathrm{a}}=e_{\mathrm{a}}{ }^{j} e^{\mathrm{b} i} A_{i j} e_{\mathrm{b}}^{\star}$, one finds that

$$
\begin{aligned}
A_{i j}= & {\left[-N^{-2}\left(\hat{\partial}_{0}\right)^{2}+\bar{\nabla}^{k} \bar{\nabla}_{k}\right] \pi_{i j} } \\
& \left.+\left[\text { other terms in } \pi_{i j} \text { and } \hat{\partial}_{0} \pi_{i j} \text { (but no second derivatives of } \pi_{i j}\right)\right],
\end{aligned}
$$

which more than hints at the fact that the tensor-index formulation of our construction involves a wave equation for $\pi_{i j}$. The tensor wave operator in (4.4) is one used by AACY.

\section{B. Taking Derivatives of the Field Equations}

The aforementioned 2-form $\Upsilon_{\mathrm{a}}$ is defined by the following equation:[]

$$
2 \Upsilon_{\mathrm{a}} \equiv \hat{D}_{0} \mathrm{G}_{\mathrm{a} \perp}-N^{\star} D^{\star} \| \mathrm{G}_{\mathrm{a}}-e_{\mathrm{ab}}^{\star} \wedge D^{\star}\left(N^{\|} \mathrm{G}^{\mathrm{b}}\right)+N a^{\mathrm{b}}\left({ }^{\star} \| \mathrm{G}_{\mathrm{b}}\right) e_{\mathrm{a}}^{\star}-D\left(N \mathrm{~T}_{\mathrm{a}}\right)
$$

(the unimportant factor of 2 sitting before $\Upsilon_{\mathrm{a}}$ is chosen only for later convenience). Here $T_{\mathrm{a}}$ is a certain carefully designed 1-form which is built purely from torsion (and spatial derivatives

\footnotetext{
${ }^{6}$ We could, of course, work instead with the slightly different operator $\hat{\square}^{\prime} \equiv-\left(N^{-1} \hat{D}_{0}\right)^{2}+\Delta$, but we shall stick with 4.3 ).

${ }^{7}$ See the Eq. (5.4) for the tensor-index definition corresponding to $\Upsilon_{\mathrm{a}}$.
} 
of torsion) whose particular form [given in the appendix Eq. (C6)] need not concern us yet. Notice that $\Upsilon_{\mathrm{a}}$ has been constructed by taking a "time derivative" of the equation of motion (3.14 c), with certain spatial derivatives of the momentum and rotation constraints subtracted from the result. Let us briefly comment on the origin of the various terms in the definition (4.5). In performing the $3+1$ decomposition of $\hat{D}_{0} \mathrm{G}_{\mathrm{a} \perp}$, one generates certain Einstein 3-form and torsion terms, in addition to other terms which are built with neither Einstein 3-form nor the residual torsion. Now, in fact, the subsequent terms in $\Upsilon_{\mathrm{a}}$ have been tailored to exactly cancel these generated terms. However, we note that our expression for $\Upsilon_{\mathrm{a}}$ is not unique, as one may always add terms to $\Upsilon_{\mathrm{a}}$ which are homogeneous in the Einstein 3 -form and torsion. Indeed, the fourth term in (4.5), linear in the undifferentiated Einstein 3-form, may be striken from the definition. We have included this term in order to obtain a more pleasing result for $\Upsilon_{\mathrm{a}}$ 's $3+1$ decomposition. However, this would seem to amount only to a matter of taste.

To obtain the $3+1$ decomposition of 2 -form (4.5), perform $3+1$ decomposition on the very first term $\hat{D}_{0} \mathrm{G}_{\mathrm{a} \perp}$ in the definition. With the splitting result (3.14 c), a straightforward expansion gives

$$
\begin{aligned}
\hat{D}_{0} \mathrm{G}_{\mathrm{a} \perp}= & -2 \hat{D}_{0}\left(N^{-1} \hat{D}_{0} \pi_{\mathrm{a}}\right)+\hat{D}_{0}\left[N^{-1} e_{\mathrm{ab}}^{\star} \wedge D\left(N a^{\mathrm{b}}\right)\right]-\hat{D}_{0} R_{\mathrm{a}} \\
& +2\left(\partial_{0} H\right) \pi_{\mathrm{a}}+2 H \hat{D}_{0} \pi_{\mathrm{a}}+\hat{D}_{0}\left(2^{\star} \pi_{\mathrm{bc}}{ }^{\star} \pi^{\mathrm{cb}} e_{\mathrm{a}}^{\star}-H^{2} e_{\mathrm{a}}^{\star}-4^{\star} \pi_{\mathrm{ba}} \pi^{\mathrm{b}}\right) .
\end{aligned}
$$

The second and third terms on the right-hand side of this identity are the ones which require some work. The third term, $-\hat{D}_{0} R_{\mathrm{a}}$, is handled in Appendix $\mathrm{C}$; therefore, focus attention on the second term. Using the evolution rule (1.11) for the cotriad and the commutator result (1.14), we write this term as follows:

$$
\begin{aligned}
\hat{D}_{0}\left[N^{-1} e_{\mathrm{ab}}^{\star} \wedge D\left(N a^{\mathrm{b}}\right)\right]= & -N a_{\mathrm{d}}\left(\Re_{\perp}^{\mathrm{bd}}+2 a^{[\mathrm{b}} K^{\mathrm{d}]}\right) \wedge e_{\mathrm{ab}}^{\star}+\epsilon_{\mathrm{abc}} K^{\mathrm{b}} \wedge D\left(N a^{\mathrm{c}}\right) \\
& -N^{-2}\left(\partial_{0} N\right) e_{\mathrm{ab}}^{\star} \wedge D\left(N a^{\mathrm{b}}\right)+N^{-1} e_{\mathrm{ab}}^{\star} \wedge D \hat{D}_{0}\left(N a^{\mathrm{b}}\right) .
\end{aligned}
$$

At this point, we perform some permutation-symbol gymnastics with the first term on the right-hand side in order to reach

$$
\begin{aligned}
& \hat{D}_{0}\left[N^{-1} e_{\mathrm{ab}}^{\star} \wedge D\left(N a^{\mathrm{b}}\right)\right]= \\
& \quad N a_{\mathrm{a}} G_{\perp} \mathrm{b} e_{\mathrm{b}}^{\star}-\frac{1}{2} \epsilon_{\mathrm{abc}} N\left(\Re^{\mathrm{bc}}{ }_{\perp}+2 a^{\mathrm{b}} K^{\mathrm{c}}\right) \wedge a+N a_{\mathrm{a}} a_{\mathrm{b}}\left(H \delta^{\mathrm{bc}}-K^{\mathrm{bc}}\right) e_{\mathrm{c}}^{\star} \\
& \quad+\epsilon_{\mathrm{abc}} K^{\mathrm{b}} \wedge D\left(N a^{\mathrm{c}}\right)-N^{-2}\left(\partial_{0} N\right) e_{\mathrm{ab}}^{\star} \wedge D\left(N a^{\mathrm{b}}\right)+N^{-1} e_{\mathrm{ab}}^{\star} \wedge D D_{0}\left(N a^{\mathrm{b}}\right) .
\end{aligned}
$$

Next, with the index evolution rule (1.12) we derive the identity

$$
\hat{D}_{0}\left(N a^{\mathrm{b}}\right) \equiv \hat{D}_{0}\left(e^{\mathrm{b} i} \partial_{i} N\right)=D^{\mathrm{b}}\left(\partial_{0} N\right)+N^{2} a_{\mathrm{d}} K^{\mathrm{db}}
$$

(on a scalar like $\partial_{0} N$, the action of $D^{\mathrm{b}}$ is $e^{\mathrm{b} i} \partial_{i}$ ), and then insert it into the last term of (4.8), expanding the resulting expression. At the same time, we make a substitution into

\footnotetext{
${ }^{8} \mathrm{We}$ note that there is another possible route which one could take at this point. Namely, one could directly substitute the appendix result (B4) into the first term of (4.7). If one intends to follow this route, then one should first strike the term $N a^{\mathrm{b}}\left({ }^{\star} \|_{\mathrm{b}}\right) e_{\mathrm{a}}^{\star}$ from the definition (4.5) of $\Upsilon_{\mathrm{a}}$.
} 
the second term in (4.8) with the appendix result (C7). Combining the result of these endeavors with (4.6) and the appendix expression ( desired expression for $\hat{D}_{0} \mathrm{G}_{\mathrm{a} \perp}$. Finally, all extrinsic curvature terms in the expression are re-expressed in terms of $\pi^{\mathrm{ab}}$ via (3.8).

With the splitting result for $\hat{D}_{0} G_{a}$, we express the $3+1$ decomposition of the 2 -form (4.5) as follows:

$$
\Upsilon_{\mathrm{a}}=N \hat{\square} \pi_{\mathrm{a}}+U_{\mathrm{a}}^{\pi}+U_{\mathrm{a}}^{T}+V_{\mathrm{a}}
$$

where explicitly one has the source term

$$
\begin{aligned}
U_{\mathrm{a}}^{\pi}= & \hat{D}_{0}\left({ }^{\star} \pi_{\mathrm{bc}}{ }^{\star} \pi^{\mathrm{cb}} e_{\mathrm{a}}^{\star}-\frac{1}{2} H^{2} e_{\mathrm{a}}^{\star}-2^{\star} \pi_{\mathrm{ba}} \pi^{\mathrm{b}}\right)+\left(\partial_{0} H\right) \pi_{\mathrm{a}}-2 N a \wedge{ }^{\star} D^{\star} \pi_{\mathrm{a}} \\
& +N a_{\mathrm{a}} a_{\mathrm{b}}{ }^{\star} \pi^{\mathrm{bc}} e_{\mathrm{c}}^{\star}+\epsilon_{\mathrm{abc}}{ }^{\star} \pi^{\mathrm{b}}{ }_{\mathrm{d}} D\left(N a^{\mathrm{c}} e^{\mathrm{d}}\right)+2 \epsilon_{\mathrm{abc}} N^{-1} D\left(N^{2 \star} \pi_{\mathrm{d}}{ }^{\mathrm{b}} a^{(\mathrm{c}} e^{\mathrm{d})}\right) \\
& -N^{-1} e_{\mathrm{ab}}^{\star} \wedge D\left(N^{2} H a^{\mathrm{b}}\right)+N^{-2} f \hat{D}_{0} \pi_{\mathrm{a}}-\frac{1}{2} N^{-2} f e_{\mathrm{ab}}^{\star} \wedge D\left(N a^{\mathrm{b}}\right),
\end{aligned}
$$

the torsion term

$$
U_{\mathrm{a}}^{T}=\frac{1}{4} \delta_{\mathrm{ab}} N^{2}\left[{ }^{\star} d\left(N^{-1} T\right)\right] a \wedge e^{\mathrm{b}}-\frac{1}{2} \delta_{\mathrm{ab}} N^{2} a \wedge{ }^{\star} D^{\star}\left(N^{-1} e^{\mathrm{b}} \wedge{ }^{\star} T\right)+\frac{1}{2} N^{-1} D\left(N^{2} a_{\mathrm{a}}{ }^{\star} T\right),
$$

and the slicing term

$$
V_{\mathrm{a}}=\frac{1}{2} N^{-1} e_{\mathrm{ab}}^{\star} \wedge D\left(D^{\mathrm{b}} f\right)
$$

In these expression, we have defined $f \equiv \partial_{0} N+N^{2} H$. Notice that all of the secondderivative terms in (4.10) involving $\pi_{\mathrm{a}}$ (other than those found in $\hat{\square} \pi_{\mathrm{a}}$ ) have been collected together in the slicing term (4.13). Also, make note of the torsion appearing in $U_{\mathrm{a}}^{T}$. Here we consider $T$ as a short-hand for $2 e^{\mathrm{a}} \wedge{ }^{\star} \pi_{\mathrm{a}}$ [cf. Eq. (3.10)]; and, therefore, in effect we have the combined expression $U_{\mathrm{a}} \equiv U_{\mathrm{a}}^{\pi}+U_{\mathrm{a}}^{T}$ as the full source term. We would, of course, prefer to incorporate some or all of $U_{\mathrm{a}}^{T}$ into the definition of (4.5) in order to obtain a more compact expression for the source term. Unfortunately, as we shall soon argue, it would seem that this is not permissible, as doing so would destroy the desired equivalence with Einstein's theory. However, it is possible to re-express the combined source term in such a way that the residual torsion 2 -form is not so manifestly apparent. Indeed, following the alternative route mentioned in the footnote before Eq. (4.8), one finds a combined source term which is almost entirely "torsion-free." We have chosen the route that we have, because it happens to lead to more aesthetically pleasing equations than does the alternative. That is to say, only exact exterior derivatives appear in the combined source term as we have written it. Following the other route, one finds a lot of triad covariant derivatives, $D_{\mathrm{b}}$ 's, surfacing in the expressions. To our mind, the chosen route is more in spirit with the DF language intended to be showcased here.

\section{Wave-equation Formulation of General Relativity}

For the vanishing of $\Upsilon_{\mathrm{a}}$ to produce a good wave-equation, the term $V_{\mathrm{a}}$ must be equal to a 2-form which involves fewer than second derivatives of $H$. As described by AACY, there 
appears to be a number of ways to do this. For our purposes it suffices to enforce the simple harmonic slicing condition, [4]

$$
f \equiv \partial_{0} N+N^{2} H=0 .
$$

(An obvious generalization of this equation is obtained by assuming that $f=f\left(t, x^{i}\right)$ is a prescribed well-behaved function.) One immediately sees that $V_{\mathrm{a}}$ vanishes for a harmonic time-slicing. The vanishing of $V_{\mathrm{a}}$ also removes the term involving triple derivatives of the lapse. Further, two terms in the expression (4.11) for $U_{\mathrm{a}}^{\pi}$ also vanish for harmonic timeslicing.

As the new Einstein system of equations of motion, we now offer

$$
\begin{aligned}
\hat{D}_{0} e_{\mathrm{a}}^{\star} & =-2 N \pi_{\mathrm{a}} \\
\hat{\square}_{\pi_{\mathrm{a}}} & =-N^{-1} U_{\mathrm{a}} \\
\partial_{0} N & =-N^{2} H .
\end{aligned}
$$

The appropriate Cauchy data on the initial slice are $e_{\mathrm{a}}^{\star}, \pi_{\mathrm{a}}, N>0$, and $\hat{D}_{0} \pi_{\mathrm{a}}$ subject to the following constraints:

$$
\begin{aligned}
& 0=-4 \pi^{[\mathrm{ab}]} \\
& 0=-2 D \pi_{\mathrm{a}} \\
& 0=2^{\star} \pi_{\mathrm{ab}} \pi^{\mathrm{ba}}-H \pi^{\mathrm{b}}{ }_{\mathrm{b}}+e^{\mathrm{a}} \wedge R_{\mathrm{a}} \\
& 0=-2 N^{-1} \hat{D}_{0} \pi_{\mathrm{a}}+N^{-1} e_{\mathrm{ab}}^{\star} \wedge D\left(N a^{\mathrm{b}}\right)-R_{\mathrm{a}}-H^{2} e_{\mathrm{a}}^{\star}+{ }^{\star} \pi_{\mathrm{bc}}{ }^{\star} \pi^{\mathrm{cb}} e_{\mathrm{a}}^{\star}+2 H \pi_{\mathrm{a}}-4^{\star} \pi_{\mathrm{ba}} \pi^{\mathrm{b}},
\end{aligned}
$$

where the understanding is that these constraint equations are pulled back to the initial-data surface. Notice that we are requiring the full Einstein 3-form to vanish on the initial data surface. Both the shift vector $\beta^{j}$ and the skew-symmetric rotation matrix $\phi_{\text {ab }}$ play a passive role in our formalism, as they have been absorbed into the operator $\hat{D}_{0}$.

\section{VERIFICATION OF THE ORIGINAL EINSTEIN EQUATIONS}

Before showing that the new system of equations is equivalent to the original Einstein equations, we should demonstrate that it is indeed solvable. However, for sake of brevity, we only note here that the treatment of this issue found in the third reference of [4] goes through essentially unaltered for the case at hand. Therefore, let us turn to the proof of equivalence.

CLAIM: The vanishing of the Einstein 3-form and torsion is equivalent to the 2 -form equation

$$
\Upsilon_{\mathrm{a}}=0,
$$

when subject to the initial-value conditions

$$
\begin{aligned}
& \|\left.\mathrm{G}_{\perp}\right|_{\Sigma}=0 \\
& \left.{ }^{\|} \mathrm{G}_{\mathrm{a}}\right|_{\Sigma}=0 \\
& \left.\mathrm{G}_{\mathrm{a} \perp}\right|_{\Sigma}=0 \\
& \left.T\right|_{\Sigma}=0 \text {. }
\end{aligned}
$$

Here $\left.\right|_{\Sigma}$ means "pull-back to the initial-data surface." 


\section{A. Method of Proof and Two Lemmas}

To prove the claim, it proves most convenient to switch to and proceed in index notation. Our proof follows the arguments of AACY and of York 90 quite closely. We shall have need of the spacetime covariant derivative which "sees" quasi-coordinate indices, and we use $\nabla_{\mu}$ to denote this covariant derivative. Consider the action of $\hat{\partial}_{0}$ and $\bar{\nabla}_{i}$ on $\Sigma$ scalars (such as $G_{00}$ ), $\Sigma$ 1-forms (such as $G_{0 i}$ ), and $\Sigma 2$-index tensors (such as $G_{i j}$ ) which arise from the various components of a 2-index spacetime tensor (such as $G_{\alpha \beta}$ ). The proof is facilitated by relating the action of $\hat{\partial}_{0}$ and $\bar{\nabla}_{i}$ on these $\Sigma$ objects to the action of $\nabla_{0}$ and $\nabla_{i}$ on the same objects. Such relations follow from the definition of these operators and the values of the connection coefficients associated with the quasi-coordinate frame (1.1), those being given by

$$
\Gamma_{\lambda \mu}^{\sigma}=\frac{1}{2} g^{\sigma \kappa}\left(e_{\lambda}\left[g_{\kappa \mu}\right]+e_{\mu}\left[g_{\kappa \lambda}\right]-e_{\kappa}\left[g_{\lambda \mu}\right]\right)+\frac{1}{2}\left(g^{\sigma \kappa} g_{\mu \nu} C_{\kappa \lambda}{ }^{\nu}+g^{\sigma \kappa} g_{\lambda \nu} C_{\kappa \mu}{ }^{\nu}-C_{\lambda \mu}{ }^{\sigma}\right) .
$$

Here the $C_{\mu \nu}{ }^{\lambda} \equiv\left[e_{\mu}, e_{\nu}\right]^{\lambda}$ are the structure functions associated with the frame (1.1). The frame choice (1.1) ensures that the $\bar{\Gamma}_{j k}^{i} \equiv \Gamma_{j k}^{i}$ are the ordinary Christoffel symbols associated with the $\Sigma$ coordinate frame. Throughout the proof, we shall employ the short-hand (l.h.E.T. $)_{n}$ to mean "additive remainder terms linear and homogeneous in both the Einstein tensor and the torsion tensor and their derivatives of order $\leq n$." When remainder terms are built solely with the Einstein tensor or solely with the torsion tensor, we shall employ the short-hands (1.h.E.) ${ }_{n}$ and (1.h.T.) ${ }_{n}$, whose meanings should be clear.

We begin by defining a $\Sigma$ tensor $\Upsilon_{i j}$ via $\Upsilon_{\mathrm{a}}=e_{\mathrm{a}}{ }^{j} e^{\mathrm{b} i} \Upsilon_{i j} e_{\mathrm{b}}^{\star}$. Provided that the triad is nowhere degenerate, the vanishing of $\Upsilon_{i j} \Longleftrightarrow$ the vanishing of $\Upsilon_{\mathrm{a}}$. Direct calculation now yields

$$
\Upsilon_{i j}=-\hat{\partial}_{0} G_{i j}+\bar{\nabla}_{i} G_{0 j}+\bar{\nabla}_{j} G_{0 i}-h_{i j} \bar{\nabla}^{l} G_{0 l}-C_{i j}+(\text { l.h.E. })_{0} .
$$

Hence, in (5.4) no derivatives of the Einstein tensor appear other than those explicitly shown. The $\Sigma$ tensor $C_{i j}$ is defined via $e_{\mathrm{a}}{ }^{j} e^{\mathrm{b} i} C_{i j} e_{\mathrm{b}}^{\star}=D\left(N \mathrm{~T}_{\mathrm{a}}\right)$, and for it we find the following explicit expression [cf. the footnote before (C6)]:

$$
C_{i j}=\frac{1}{2} \bar{\nabla}^{l}\left\{N^{2}\left[\bar{\nabla}_{i}\left(N^{-1} T_{j l}\right)+\bar{\nabla}_{j}\left(N^{-1} T_{i l}\right)+\bar{\nabla}_{l}\left(N^{-1} T_{i j}\right)\right]\right\} .
$$

The $i \leftrightarrow j$ symmetry present in the first two terms proves to be quite important for our construction. In fact, this symmetry (in tandem with the way the particular powers of the lapse enter the expression), ensures that the antisymmetric piece of $N^{-1} C_{i j}$ is the Laplacian on $T_{i j}$, up to a trivial (1.h.T.) $)_{0}$ term. Compare the definition (5.4) of $\Upsilon_{i j}$ with AACY's definition of $\Omega_{i j}$ (or, even better, with their $\Omega_{i j}^{\prime}$ ). In this comparison, be sure to note that both $\hat{\partial}_{0} G_{i j}$ and $C_{i j}$ are not manifestly symmetric in our formalism.

With the aforementioned relations between the actions of $\hat{\partial}_{0}$ and $\bar{\nabla}_{i}$ and of $\nabla_{0}$ and $\nabla_{i}$, we write

$$
\Upsilon_{i j}=-\nabla_{0} G_{i j}+\nabla_{i} G_{0 j}+\nabla_{j} G_{0 i}-h_{i j} \nabla^{k} G_{0 k}-C_{i j}+(\text { l.h.E. })_{0}
$$

and, moreover, we have the following two lemmas for $C_{i j}$. 
LEMmA 1: The expression for $C_{i j}$ may be written as

$$
C_{i j}=\frac{1}{2} N\left[\nabla^{l} \nabla_{i} T_{j l}+\nabla^{l} \nabla_{j} T_{i l}+\nabla^{l} \nabla_{l} T_{i j}\right]+\lambda_{i j}+(\text { l.h.E.T. })_{0},
$$

where $\lambda_{i j}$ is a symmetric remainder term of (l.h.T.) $)_{1}$ order.

To prove the lemma simply expand the expression (5.5) for $C_{i j}$. Then the proof reduces to verifying that $\nabla_{k} \nabla_{l} T_{i j}=\bar{\nabla}_{k} \bar{\nabla}_{l} T_{i j}+$ (l.h.E.T. $)_{0}$. Now, viewed as a spacetime tensor, the torsion $T_{\alpha \beta}$ is tangential to the $\Sigma$ slices $\left(T_{0 i}=T_{00}=0\right)$. It follows that $\nabla_{k} T_{i j}=\bar{\nabla}_{k} T_{i j}$; and, hence, the only troublesome terms which arise in obtaining the verification above are those of the form $\nabla_{0} T_{i j}$. But such terms may be eliminated in favor of (1.h.E.T.) ${ }_{0}$ ones via (2.9) [technically, via (2.9) as we write it later in $(5.13 \mathrm{c})]$.

LEMmA 2: The tensor $C_{i j}$ obeys the property $\nabla^{i} C_{i j}=(\text { l.h.T. })_{1}$.

Viewed as a spacetime tensor, $C_{\alpha \beta}$ is by definition purely spatial $\left(C_{0 i}=C_{i 0}=C_{00}=0\right)$; it follows that $\nabla^{i} C_{i j}=\bar{\nabla}^{i} C_{i j}$. With this fact, one can prove the lemma directly by forming $\bar{\nabla}^{i} C_{i j}$ and making appeals to the Ricci identity. Alternatively, with the definition of $C_{i j}$ one may show that

$$
e_{\mathrm{a}}{ }^{j} \bar{\nabla}^{i} C_{i j}={ }^{\star} D^{2}\left(N \mathrm{~T}_{\mathrm{a}}\right) .
$$

Therefore, since $T_{\mathrm{a}}$ is (l.h.T.) $)_{1}$ by construction, the Cartan identity for the creation of curvature (symbolically $D^{2}=R \wedge$ ) yields the result (provided a non-degenerate triad).

\section{B. Zero-order Wave Equation for the Torsion}

Before turning to the main part of the proof, let us derive a certain zero-order wave equation for the residual torsion tensor $T_{i j}$ which is implicitly defined by the vanishing of $\Upsilon_{i j}$. This derivation will also further elucidate the precise role played in our formalism by the 1 -form $T_{\mathrm{a}}$ appearing in the definition (4.5). The form of (5.7) ensures that the antisymmetric piece of $C_{i j}$ obeys

$$
C_{[i j]}=\frac{1}{2} N \nabla^{l} \nabla_{l} T_{i j}+(\text { l.h.E.T. })_{0},
$$

Note that the extra terms in this equation are linear and homogeneous in the Einstein and torsion tensors with no spatial derivatives of them appearing. In particular, the tensor $\lambda_{i j}$ has been killed by the antisymmetrization.

Now consider the index version of (2.9), which may be expressed as

$$
\nabla_{0} T_{i j}-2 N G_{[i j]}=(\text { l.h.T. })_{0} .
$$

With Eq. (5.9) in mind, we apply $\nabla^{0}$ [the same as applying $N^{-2} \nabla_{0}$ for our frame (1.1)] to equation (5.10) and expand. Along the way, we make further substitutions with (5.10) to replace $\nabla_{0}$ (l.h.T. $)_{0}$ terms with (l.h.E.T.) $)_{0}$ ones. We then reach

$$
\nabla^{0} \nabla_{0} T_{i j}-2 N^{-1} \nabla_{0} G_{[i j]}=(\text { l.h.E.T. })_{0} .
$$

Next, substituting (5.6) into (5.11) and appealing to (5.9), we obtain 


$$
\square T_{i j}=-2 N^{-1} \Upsilon_{[i j]}+(\text { l.h.E.T. })_{0},
$$

with $\square$ denoting the standard covariant D' Alembertian $\nabla_{\mu} \nabla^{\mu}$. Eq. (5.12) shows that the vanishing of $\Upsilon_{i j}$ yields a zero-order wave equation for the residual torsion 2 -form. This zero-order character proves to be quintessential in establishing equivalence between our DF system of equations given above and the vacuum Einstein equations. In fact, we have rigged the definition [cf. Eqs. (C5) and (C6)] of $\mathrm{T}_{\mathrm{a}}$ in (4.5) to ensure this zero-order character for (5.12). Note that there is a projection into $\Sigma$ implicit in (5.12). That is to say, while $T_{0 i}=0$ for the torsion viewed as a spacetime tensor $T_{\alpha \beta}$, it is not the case that $\square T_{0 i}$, the tangentialnormal piece of $\square T_{\alpha \beta}$, need be zero (of course, $\square T_{00}$ does indeed vanish by antisymmetry). Eq. (5.12) has nothing to say about $\square T_{0 i}$; however, for our purposes this observation is inconsequential.

\section{Twice-contracted Bianchi Identities and Equivalence Proof}

We shall require the so-called twice-contracted Bianchi identities $\nabla^{\mu} G_{\mu \nu}=0$ (index ordering is important!), which we expand out,

$$
\begin{aligned}
& 0=\nabla^{0} G_{00}+\nabla^{j} G_{j 0} \\
& 0=\nabla^{0} G_{0 i}+\nabla^{j} G_{j i} .
\end{aligned}
$$

As is well-known, these identities are related to the preservation in time of the Hamiltonian and momentum constraints. Likewise, our identity (5.10), which we conveniently write now as

$$
0=\nabla_{0} T_{i j}+(\text { l.h.E.T. })_{0},
$$

is derived from the Bianchi identity for the torsion and is related to the preservation in time of the rotation constraint.

As the first step in the proof, form $\nabla^{i} \Upsilon_{i j}$, use the Ricci identity to commute covariant derivatives, insert $5.13 \mathrm{~b}$ ), and appeal to the second lemma, in order to obtain

$$
\square G_{0 j}=(\text { l.h.E.T. })_{1} .
$$

As the second step, apply $\square$ to the identity (5.13/a). Repeated use of the Ricci identity on the resulting expression yields

$$
\nabla^{0} \square G_{00}+\nabla^{j} \square G_{j 0}=(\text { l.h.E. })_{1} .
$$

At this point, we insert the result $G_{j 0}=G_{0 j}+T_{j k} \nabla^{k} N$ [cf. Eq. (2.14)]. Then, appeals to the earlier findings (5.12) and (5.14) show that

$$
\nabla_{0} \square G_{00}=(\text { l.h.E.T })_{2} .
$$

Finally, as the proof's third step, apply $\square$ to the definition of $N^{-1} \Upsilon_{i j}$ (which vanishes) as given in (5.6), expand out the resulting expression, repeatedly use the Ricci identity, and make several appeals to (5.14), thereby arriving at 


$$
N^{-1} \nabla_{0} \square G_{i j}+\square\left(N^{-1} C_{i j}\right)=(\text { l.h.E.T. })_{2} .
$$

The lapse factor has been included to remove the lapse term in $C_{i j}$ [cf. the form of this tensor given in the first lemma, Eq. (5.7)]. Note that $\square\left(N^{-1} C_{i j}\right)$ is (l.h.T.) $)_{4}$; however, that it is also (l.h.E.T.) $)_{2}$ may be shown by the following argument which makes use of (5.7). For the leading terms in $N^{-1} C_{i j}$, those quadratic in the covariant derivatives, we can move the action of the $\square$ onto the torsion tensors in the expression. In so moving the $\square$, we must again repeatedly use the Ricci identity, a process which generates only byproduct (l.h.T. $)_{2}$ terms. For $\square\left(N^{-1} \lambda_{i j}\right)$, a priori (1.h.T. $)_{3}$, we note that, again up to (1.h.T. $)_{2}$ terms, the $\square$ can be moved onto the torsion tensors in the expression. Therefore, invoking (5.12), we get

$$
\nabla_{0} \square G_{i j}=(\text { l.h.E.T. })_{2} .
$$

Notice that the application of $\square$ has mapped (1.h.T) $)_{2}$ terms into (1.h.E.T) $)_{2}$ ones. The ability to take this crucial step rests squarely on the zero-order character of the wave equation (5.12).

With (5.14), (5.16), (5.18), we obtain the following system of equations:

$$
\begin{aligned}
\hat{\partial}_{0} \square G_{0 j} & =(\text { l.h.E.T. })_{2} \\
\hat{\partial}_{0} \square G_{00} & =(\text { l.h.E.T. })_{2} \\
\hat{\partial}_{0} \square G_{i j} & =(\text { l.h.E.T. })_{2} \\
\hat{\partial}_{0} T_{i j} & =(\text { l.h.E.T. })_{0} .
\end{aligned}
$$

This system is (up to homogeneous Einstein-tensor and torsion-tensor terms) essentially the system considered in the works of AACY, although here we see the novel appearance of the torsion tensor. It is a strictly hyperbolic system. All (l.h.E.T.) $)_{2}$ and (l.h.E.T.) $)_{0}$ terms on the right-hand side may be shown to be zero on the initial Cauchy surface by (i) the (index versions of the) boundary conditions (5.2), (ii) the Bianchi identities (5.13), (iii) the vanishing of $\Upsilon_{i j}$, and (iv) derivatives of the previous three items. We infer that $T_{i j}$ and all pieces of the Einstein tensor save $G_{j 0}$ vanish in the future domain of dependence $D^{+}(\Sigma)$. We then get the vanishing of $G_{j 0}$ from the vanishing of $G_{0 j}$ and $T_{i j}$ by the identity (2.14). Equivalence has thus been established.

\section{CONCLUSION}

We conclude by giving some basic instructions (with cautionary remarks) for rewriting our DF results in the tensor-index language. At the outset, we must admit that we find passing back and forth between the DF and tensor-index versions of wave-equation general relativity to be not as clean and direct as we had originally hoped would be the case. Passage to the tensor-index version of the formalism essentially amounts to (i) defining $\Upsilon_{i j}$ as before in Eq. (5.4) and then (ii) simply setting the torsion to zero by hand in all expressions [hence $C_{i j}=0$ in (5.4)], thereby obtaining a symmetric $\Upsilon_{i j}$. Now, it is tempting to simply use $\Upsilon_{i j}$ as the jumping-off point for deriving an tensor-index wave equation for $\pi_{i j}$, working with $\Upsilon_{i j}$ in the same fashion that AACY work with their $\Omega_{i j}$. However, as we shall now argue, though this is essentially the correct procedure, one must be careful to extract superfluous Einstein 
tensor terms which are indeed present in $\Upsilon_{i j}$. In (5.4) these superfluous terms have simply been swept into the (1.h.E.) $)_{0}$ short-hand, but let us now examine the origin and nature of these terms in more detail.

Consider again the source term (4.11) written as follows: $U_{\mathrm{a}}^{\pi}=e_{\mathrm{a}}^{j} e^{\mathrm{b} i} U_{i j}^{\pi} e_{\mathrm{b}}^{\star}$. Now, in fact, $U_{\mathrm{a}}^{\pi}$ still has some Einstein tensor "buried in it," i. e. one finds that $U_{i j}^{\pi}$ contains contracted covariant derivatives of $\pi_{i j}$, e. g. $\bar{\nabla}_{l} \pi_{i}^{l}$, coming from the sixth term in the expression for $U_{\mathrm{a}}^{\pi}$. But $2 \bar{\nabla}_{l} \pi_{i}^{l}=G_{\perp i}$, and, hence, in the $3+1$ decomposition of $\Upsilon_{i j}$ superfluous terms involving the momentum constraint $G_{\perp i}$ arise. Therefore, one should locate and remove these superfluous momentum-constraint terms in the passage to the tensor-index formalism. However, besides such superfluous momentum-constraint terms, another superfluous Einstein-tensor term also arises in the passage to the tensor-index version of the formalism. Indeed, consider the leading term in $\Upsilon_{\mathrm{a}}$, namely,

$$
\hat{D}_{0} \mathrm{G}_{\mathrm{a} \perp}=-e_{\mathrm{a}}^{j} e^{\mathrm{b} i}\left(\hat{\partial}_{0} G_{i j}\right) e_{\mathrm{b}}^{\star}-G_{i j} \hat{D}_{0}\left(e_{\mathrm{a}}^{j} e^{\mathrm{b} i} e_{\mathrm{b}}^{\star}\right) .
$$

The expansion (6.1) shows that our $\Sigma$ tensor $\Upsilon_{i j}$ has an undifferentiated $G_{i j}$ term "buried" in it; hence, in passing to the tensor-index language we be should be careful to locate and remove this term.

One might ask, why not simply work with a modified definition of $\Upsilon_{\mathrm{a}}$ (call it $\Upsilon_{\mathrm{a}}^{\prime}$ ) such that passage to the tensor-index formalism is rather more direct, i. e. no Einstein tensors surface in the $3+1$ decomposition of $\Upsilon_{i j}^{\prime}$ (obtained from $\Upsilon_{\mathrm{a}}^{\prime}=e_{\mathrm{a}}{ }^{j} e^{\mathrm{b} i} \Upsilon_{i j}^{\prime} e_{\mathrm{b}}^{\star}$ ). However, the differential-form wave equation for $\pi_{\mathrm{a}}$ obtained from the corresponding 2-form equation $\left(\Upsilon^{\prime}\right)$ is quite a bit messier than the one we have considered, and, if fact, this wave equation contains superfluous Einstein 3-form terms in it! Therefore, if the intent is to remain within the framework of DF, then we find it preferable to work with (5.1) and its $3+1$ decomposition as given. However, this means that one must not be too naive when rewriting the results in the tensor-index language. One must be careful to extract superfluous Einstein-tensor terms which will arise in the tensor equations directly obtained from the corresponding DF equations. The non-triviality associated with going back and forth between the two languages can be traced to whether it is the Einstein 3-form or the Einstein tensor which is held as the fundamental object (i. e. which is derived from which).

\section{ACKNOWLEDGMENTS}

Many thanks to both A. Anderson and J. W. York for guidance concerning several aspects of this work. J. W. York provided me with an early copy of his personal notes on the original (tensor-index) hyperbolic formulation of general relativity. These notes proved quintessential in the development of the ideas presented here. I am also grateful for comments by and discussions with H. Balasin and R. Beig. This research has been supported by the "Fonds zur Förderung der wissenschaftlichen Forschung" in Austria (Lise Meitner Fellowship M-00182-PHY and FWF Project 10.221-PHY). 


\section{APPENDIX A: GAUSS-CODAZZI-MAINARDI EQUATIONS WITH TORSION}

The GCM equations (see for example [1, 12]) are integrability criteria which relate the $\mathcal{M}$ Riemann tensor $\Re_{\beta \mu \nu}^{\alpha}$ to the $\Sigma$ Riemann tensor $R_{j k l}^{i}$, the $\Sigma$ extrinsic curvature tensor $K_{i j}$, and other geometric objects associated with the $\Sigma$ foliation. The form of these equations is complicated quite a bit by the presence of spacetime torsion. We shall present equations valid only for the limited type of spacetime torsion considered in this work.

To derive the needed equations, one may proceed in a number of ways. Perhaps the best method is to apply the form decompositions (1.3) and (1.4) to the second Cartan structure equation for $\Re_{B}^{A}$, the curvature 2 -form. This straightforward method leads to the following expressions for the time-gauge components of the $\mathcal{M}$ Riemann tensor:

$$
\begin{aligned}
\Re_{\mathrm{abcd}} & =R_{\mathrm{abcd}}+K_{\mathrm{ac}} K_{\mathrm{bd}}-K_{\mathrm{ad}} K_{\mathrm{bc}} \\
\Re_{\perp \mathrm{abc}} & =D_{\mathrm{b}} K_{\mathrm{ac}}-D_{\mathrm{c}} K_{\mathrm{ab}} \\
\Re_{\mathrm{ab} \perp \mathrm{c}} & =N^{-1} e_{\mathrm{c}}{ }^{j} \hat{\partial}_{0} \omega_{\mathrm{ab} j}-a_{\mathrm{a}} K_{\mathrm{bc}}+a_{\mathrm{b}} K_{\mathrm{ac}}-N^{-1}\left(e_{\mathrm{c}}\left[\Gamma_{\mathrm{ab} 0}\right]-\Gamma_{\mathrm{db} 0} \omega^{\mathrm{d}}{ }_{\mathrm{ac}}-\Gamma_{\mathrm{ad} 0} \omega^{\mathrm{d}}{ }_{\mathrm{bc}}\right) \\
\Re_{\mathrm{a} \perp \mathrm{b} \perp} & =N^{-1} e_{\mathrm{b}}{ }^{j} \hat{D}_{0} K_{\mathrm{a} j}+D_{\mathrm{b}} a_{\mathrm{a}}+a_{\mathrm{a}} a_{\mathrm{b}} .
\end{aligned}
$$

Let us express the identities (A1) in terms of $\Sigma$ coordinate indices. Since all of the terms in (A1 a,b) are tensorial, conversion of these equations into the required forms is immediate. Moreover, with the evolution rule (1.12) for the triad, conversion of the final identity (A1d) into $\Sigma$ coordinate indices is also not difficult. However, finding a suitable expression for (A1c) is more difficult. One can use the $\left(\mathcal{D} T^{\mathrm{a}}\right)_{\perp}$ piece (2.10) of the Bianchi identity (2.5) for the torsion 2-form, in order to express $\Re_{\mathrm{ab} \perp \mathrm{c}}$ as $\Re_{\perp \text { cab }}$ plus terms built from the torsion tensor $T_{\mathrm{ab}}$ and the lapse function $N$ (recall that without torsion the Riemann tensor is symmetric under such pair exchange). We obtain the following list:

$$
\begin{aligned}
\Re_{i j k l} & =R_{i j k l}+K_{i k} K_{j l}-K_{i l} K_{j k} \\
\Re_{\perp i j k} & =\bar{\nabla}_{j} K_{i k}-\bar{\nabla}_{k} K_{i j} \\
\Re_{j k \perp i} & =\bar{\nabla}_{j} K_{i k}-\bar{\nabla}_{k} K_{i j}+\frac{1}{2} N^{-1} \bar{\nabla}_{i}\left(N T_{j k}\right)+\frac{1}{2} N \bar{\nabla}_{j}\left(N^{-1} T_{k i}\right)-\frac{1}{2} N \bar{\nabla}_{k}\left(N^{-1} T_{j i}\right) \\
\Re_{i \perp j \perp} & =N^{-1} \hat{\partial}_{0} K_{i j}+K_{i}^{l} K_{l j}+\bar{\nabla}_{j} a_{i}+a_{i} a_{j},
\end{aligned}
$$

where we remind the reader that $T_{i j}=2 K_{[i j]}$, and $\bar{\nabla}_{i}$ stands for the ordinary $\Sigma$ covariant derivative. Clearly, equations (A2 b,c) exhibit the loss, due to torsion, of the pair-exchange symmetry enjoyed by Riemann curvature in the absence of torsion. However, note that (A2 d) is also not symmetric under $i \leftrightarrow j$ exchange. However, all of the terms on the righthand side of (A2 d), save the very first, are symmetric under $i \leftrightarrow j$ exchange. In particular, note that the term $\bar{\nabla}_{j} a_{i}=\bar{\nabla}_{j} \bar{\nabla}_{i} \log N$ is indeed symmetric, as we have set the $\Sigma$ torsion tensor $T_{j k}^{i}$ manifestly equal to zero. The behavior of $\Re_{i \perp j \perp}=\Re_{\perp i \perp j}$ under the pair exchange $\perp i \leftrightarrow \perp j$ is also evident from (2.7).

\section{APPENDIX B: COMMUTATOR OF $\hat{D}_{0}$ AND $D$}

To evaluate the commutator $\left[\hat{D}_{0}, D\right] \psi^{\mathrm{a}}$, where $\psi^{\mathrm{a}}=" \psi^{\mathrm{a}}$ is an arbitrary triad-valued spatial form, it suffices to consider the action of the squared spacetime exterior covariant 
derivative $\mathcal{D}^{2}$ on an arbitrary tetrad-valued spatial form $\psi^{A}={ }^{\|} \psi^{A}$. Applying the form decompositions $(1.3,1.4)$ to the expression $\mathcal{D} \psi^{A}$, one finds that

$$
\begin{aligned}
\|\left(\mathcal{D} \psi^{\perp}\right) & =d \psi^{\perp}-K_{\mathrm{b}} \wedge \psi^{\mathrm{b}} \\
\left(\mathcal{D} \psi^{\perp}\right)_{\perp} & =N^{-1} \hat{\partial}_{0} \psi^{\perp}+a_{\mathrm{b}} \psi^{\mathrm{b}} \\
\|\left(\mathcal{D} \psi^{\mathrm{a}}\right) & =D \psi^{\mathrm{a}}-K^{\mathrm{a}} \wedge \psi^{\perp} \\
\left(\mathcal{D} \psi^{\mathrm{a}}\right)_{\perp} & =N^{-1} \hat{D}_{0} \psi^{\mathrm{a}}+a^{\mathrm{a}} \psi^{\perp} .
\end{aligned}
$$

Now, a straightforward expansion yields that

$$
\begin{aligned}
\mathcal{D}^{2} \psi^{\mathrm{a}}= & D^{\|}\left(\mathcal{D} \psi^{\mathrm{a}}\right)-K^{\mathrm{a}} \wedge{ }^{\|}\left(\mathcal{D} \psi^{\perp}\right) \\
& +e^{\perp} \wedge\left\{N^{-1}\left[\hat{D}_{0} \|\left(\mathcal{D} \psi^{\mathrm{a}}\right)-D N\left(\mathcal{D} \psi^{\mathrm{a}}\right)_{\perp}\right]+a^{\mathrm{a} \|}\left(\mathcal{D} \psi^{\perp}\right)+K^{\mathrm{a}} \wedge\left(\mathcal{D} \psi^{\perp}\right)_{\perp}\right\} .
\end{aligned}
$$

However, by the second Cartan structure equation we know that the above expression is also $\mathcal{D}^{2} \psi^{\mathrm{a}}=\Re^{\mathrm{a}}{ }_{A} \wedge \psi^{A}$, where as before $\Re^{A}{ }_{B}$ is the matrix-valued curvature two-form of Cartan, which in index notation is represented by the mixed-components $\Re_{B \mu \nu}^{A}$ of the spacetime Riemann tensor. Therefore, taking the $i_{\perp}$ inner derivative of $(\mathbb{B} 2)$, inserting the relations (B1), and collecting like terms in $\psi^{\perp}$ and $\psi^{\mathrm{a}}$, we determine the following identity:

$$
\begin{aligned}
& \left(\Re^{\mathrm{a}}{ }_{\perp \perp}+N^{-1} \hat{D}_{0} K^{\mathrm{a}}+D a^{\mathrm{a}}+a^{\mathrm{a} a}\right) \wedge \psi^{\perp}= \\
& N^{-1}\left(\hat{D}_{0} D-D \hat{D}_{0}\right) \psi^{\mathrm{a}}-\left(\Re_{\mathrm{b} \perp}^{\mathrm{a}}+a^{\mathrm{a}} K_{\mathrm{b}}-a_{\mathrm{b}} K^{\mathrm{a}}\right) \wedge \psi^{\mathrm{b}} .
\end{aligned}
$$

Since each side of the above equation is independent of the other, we get two relations. The first yields the "dynamical" GCM equation (A1d). The other equation we get from (B3) is the desired one (1.14).

In the expression (1.14) the curvature term can be eliminated in favor of extrinsiccurvature and acceleration $\left(a_{\mathrm{b}}=D_{\mathrm{b}} \log N\right)$ terms. Indeed, using the GCM equation (A2 c), one may derive the following identity:

$$
\Re_{\mathrm{ab} \perp \mathrm{d}}+2 a_{[\mathrm{a}} K_{\mathrm{b}] \mathrm{d}}=N^{-1}\left[D_{\mathrm{a}}\left(N K_{(\mathrm{db})}\right)+D_{\mathrm{d}}\left(N K_{(\mathrm{ab})}\right)-D_{\mathrm{b}}\left(N K_{(\mathrm{da})}\right)-D_{\mathrm{d}}\left(N K_{\mathrm{ba}}\right)\right] .
$$

Remarkably, on the way to this result all of the torsion terms stemming from (A2c) conspire in such a way that only the symmetric piece of the extrinsic curvature tensor appears in all but the last term above. If desired, this identity may be inserted into the result (1.14) for the commutator. As an application of these considerations, consider a triad-valued 0form $f_{\mathrm{b}}=e_{\mathrm{b}}{ }^{i} f_{i}$ obtained by "soldering" the free index of a $\Sigma 1$-form $f_{i}$. With (B4) and the evolution rules (1.12) in hand, a careful examination of the expression $e^{\mathrm{b}}{ }_{j} i_{\partial_{i}}\left(\left[\hat{D}_{0}, D\right] f_{\mathrm{b}}\right)$ (note the "hook" with $\partial_{i}$ ) yields the tensor-index form of the commutator. Namely,

$$
\left[\hat{\partial}_{0}, \bar{\nabla}_{i}\right] f_{j}=\left[\bar{\nabla}_{i}\left(N K_{(j k)}\right)+\bar{\nabla}_{j}\left(N K_{(i k)}\right)-\bar{\nabla}_{k}\left(N K_{(i j)}\right)\right] f^{k} .
$$

This commutator, expanded out and written in terms of the acceleration 1-form $a_{i}=$ $\bar{\nabla}_{i} \log N$, is featured prominently in the works of AACY. 


\section{APPENDIX C: TIME DERIVATIVE OF THE TRIAD CURVATURE}

Recall that in index notation we have the following definition of the triad curvature 2form: $R_{\mathrm{a} i j} \equiv-\frac{1}{2} \epsilon_{\mathrm{abc}} R^{\mathrm{bc}}{ }_{i j}$, where the $R^{\mathrm{bc}}{ }_{i j}$ are the mixed triad-coordinate components of the $\Sigma$ Riemann tensor. We begin by using the GCM equation (A1 c) to derive the result

$$
\hat{D}_{0} R_{\mathrm{a}}=-\frac{1}{2} \epsilon_{\mathrm{abc}} D\left(N \Re^{\mathrm{bc}}+2 N a^{\mathrm{b}} K^{\mathrm{c}}\right) .
$$

This identity is essentially the $\left(\mathcal{D} \Re^{\mathrm{a}}{ }_{\mathrm{b}}\right)_{\perp}$ piece of the full (or "un-contracted") Bianchi identity $\mathcal{D} \Re_{B}^{A}=0$ for the spacetime curvature 2 -form. Indeed, another method to derive (C1) involves a $3+1$ form decomposition of the full Bianchi identity.

We now derive several auxiliary results which will be used with (C1) to get the appropriate expression for $\hat{D}_{0} R_{\mathrm{a}}$. First, using the explicit expression (3.6) for $\pi_{\mathrm{a}}$ along with the GCM equation (A1b), we obtain

$$
\frac{1}{2}\left(\Re_{\perp \mathrm{a}}^{\mathrm{bc}}\right) e_{\mathrm{bc}}^{\star}=-2^{\star} D^{\star} \pi_{\mathrm{a}}-\left(D^{\mathrm{b}} H\right) e_{\mathrm{ab}}^{\star}-\left(D^{\mathrm{b}} T_{\mathrm{a}}^{\mathrm{c}}\right) e_{\mathrm{bc}}^{\star} .
$$

Second, performing some permutation-symbol gymnastics and appealing to the identity (2.14) we find

$$
\frac{1}{2} \epsilon_{\mathrm{abc}} \Re^{\mathrm{bc}}{ }_{\perp}-\frac{1}{2}\left(\Re^{\mathrm{bc}}{ }_{\perp \mathrm{a}}\right) e_{\mathrm{bc}}^{\star}=-\left({ }^{\star} \| \mathrm{G}^{\mathrm{b}}\right) e_{\mathrm{ab}}^{\star}+\epsilon_{\mathrm{abc}} T^{\mathrm{b}}{ }_{\mathrm{d}} a^{\mathrm{d}} e^{\mathrm{c}} .
$$

Third, with the definition (3.8) we straightforwardly write

$$
\epsilon_{\mathrm{abc}} a^{\mathrm{b}} K^{\mathrm{c}}=-2 \epsilon_{\mathrm{abc}} a^{\mathrm{b} \star} \pi^{\mathrm{c}}+H a^{\mathrm{b}} e_{\mathrm{ab}}^{\star}-\epsilon_{\mathrm{abc}} T^{\mathrm{b}}{ }_{\mathrm{d}} a^{\mathrm{c}} e^{\mathrm{d}} .
$$

Notice the $c \leftrightarrow d$ antisymmetry present in the last terms of (C3) and (C4) [we have not employed the $e_{\mathrm{ab}}^{\star}$ notation in (C3) in order to highlight this antisymmetry]. Finally, we define the triad-valued 1-form $\mathrm{T}_{\mathrm{a}}$ with the seemingly strange equation

$$
-\frac{1}{2}\left(\Re_{\perp \mathrm{a}}^{\mathrm{bc}}-\Re^{\mathrm{bc}}{ }_{\perp \mathrm{a}}\right) e_{\mathrm{bc}}^{\star}=\mathrm{T}_{\mathrm{a}}+\left(D^{\mathrm{b}} T_{\mathrm{a}}^{\mathrm{c}}-a^{\mathrm{b}} T_{\mathrm{a}}^{\mathrm{c}}+\frac{1}{2} a_{\mathrm{a}} T^{\mathrm{bc}}\right) e_{\mathrm{bc}}^{\star} .
$$

We have thus defined $\mathrm{T}_{\mathrm{a}}$, because we demand that the tensor $C_{i j}$ (5.5) stemming from $\mathrm{T}_{\mathrm{a}}$ satisfies the property (5.9). This property would seem essential for the proof of equivalence given in $\S \mathrm{V}$ to go through. Although it may yet be possible to modify the definition of $\mathrm{T}_{\mathrm{a}}$ in an advantageous way, the definition that we adopt here provides us with a consistent formalism. Using the GCM splitting equations (A2 b,c), we find?

$$
\mathrm{T}_{\mathrm{a}}=\frac{1}{2} \delta_{\mathrm{ab}} N\left[{ }^{\star} d\left(N^{-1} T\right)\right] e^{\mathrm{b}}-\delta_{\mathrm{ab}} N^{\star} D^{\star}\left(N^{-1} e^{\mathrm{b}} \wedge^{\star} T\right) .
$$

as the abstract expression for this 1-form.

\footnotetext{
${ }^{9}$ Alternatively, we can write

$$
\mathrm{T}_{\mathrm{a}}=\frac{1}{4}\left[N D_{\mathrm{a}}\left(N^{-1} T^{\mathrm{bc}}\right)-2 N D^{\mathrm{b}}\left(N^{-1} T_{\mathrm{a}}^{\mathrm{c}}\right)\right] e_{\mathrm{bc}}^{\star} .
$$
}

This results allows one to easily obtain the explicit expression for $C_{i j}$ given in Eq. (5.5). 
Now add together the equations (C2), (C3), (C4), and (C5) and then perform a short calculation (taking advantage of the aforementioned $c \leftrightarrow d$ antisymmetry) in order to reach the following result:

$$
\begin{aligned}
\frac{1}{2} \epsilon_{\mathrm{abc}}\left(\Re^{\mathrm{bc}}{ }_{\perp}+2 a^{\mathrm{b}} K^{\mathrm{c}}\right)= & \mathrm{T}_{\mathrm{a}}-\left({ }^{\star} \| \mathrm{G}^{\mathrm{b}}\right) e_{\mathrm{ab}}^{\star}-2^{\star} D^{\star} \pi_{\mathrm{a}} \\
& -2 \epsilon_{\mathrm{abc}} a^{\mathrm{b} \star} \pi^{\mathrm{c}}+\left(a^{\mathrm{b}} H-D^{\mathrm{b}} H\right) e_{\mathrm{ab}}^{\star}+a_{\mathrm{a}}{ }^{\star} T .
\end{aligned}
$$

Finally, plugging ([C7) into (C1) we arrive at

$$
\begin{aligned}
\hat{D}_{0} R_{\mathrm{a}}= & -N^{\star} D^{\star \|} \mathrm{G}_{\mathrm{a}}-e_{\mathrm{ab}}^{\star} \wedge D^{\star}\left(N^{\|} \mathrm{G}^{\mathrm{b}}\right)-D\left(N \mathrm{~T}_{\mathrm{a}}\right)-2 N \Delta \pi_{\mathrm{a}}+2 N a \wedge{ }^{\star} D^{\star} \pi_{\mathrm{a}} \\
& +2 \epsilon_{\mathrm{abc}} D\left(N a^{\mathrm{b}} \pi^{\mathrm{c}}\right)+e_{\mathrm{ab}}^{\star} \wedge D\left(N H a^{\mathrm{b}}\right)-e_{\mathrm{ab}}^{\star} \wedge D\left(N D^{\mathrm{b}} H\right)-D\left(N a_{\mathrm{a}}{ }^{\star} T\right) .
\end{aligned}
$$

To get this identity, we have introduced the triad-covariant Laplacian $\Delta=\left({ }^{\star} D^{\star} D-D^{\star} D^{\star}\right)$ on triad-valued 2-forms at the price of an appeal to (3.14a). Also, in this expression we consider $T$ as a short-hand for $2 e^{\mathrm{a}} \wedge^{*} \pi_{\mathrm{a}}$ [cf. Eq. (3.10)]. We remark that other useful expressions for the final form of $\hat{D}_{0} R_{\mathrm{a}}$ are certainly possible. However, although we would, of course, prefer to incorporate the last torsional term appearing in (C8) into the definition of $\mathrm{T}_{\mathrm{a}}$ (in order to obtain a more compact expression for the final result), this would seem not to be permissible. Indeed, were we to alter the definition of $T_{a}$ in this manner, we would lose the crucial condition (5.9). More precisely, we would have to replace the (l.h.E.T. $)_{0}$ in that equation with (1.h.E.T.) $)_{1}$. This in turn would seem to spoil the proof of equivalence between our DF system of equations and the original Einstein equations. 


\section{REFERENCES}

[1] J. Isenberg and J. Nester in General Relativity and Gravitation, vol. 1, edited by A. Held (Plenum Press, New York, 1980).

[2] R. Arnowitt, S. Deser, and C. W. Misner, in Gravitation: An Introduction to Current Research, edited by L. Witten (Wiley, New York, 1962).

[3] Y. Choquet-Bruhat and J. W. York, C. R. Acad. Sci. Paris, 321, 1089 (1995).

[4] A. Abrahams, A. Anderson, Y. Choquet-Bruhat, and J. W. York, Phys. Rev. Lett. 75, 3377 (1995); A Abrahams and J. W. York, 3+1 General Relativity in Hyperbolic Form, gr-qc/9601031, January 1996; A. Abrahams, A. Anderson, Y. Choquet-Bruhat, and J. W. York, Geometrical Hyperbolic Systems for General Relativity and Gauge Theories, IFP-UNC-516, TAR-UNC-053, gr-qc/9605014, May 1996.

[5] M. Dubois-Violette and J. Madore, Commun. Math. Phys. 108, 213 (1987).

[6] J. N. Goldberg, Phys. Rev. D37, 2116 (1988).

[7] A. Ashtekar, Lectures on Non-perturbative Canonical Gravity (World Scientific Publishing Co. Pte. Ltd., Singapore, 1991).

[8] Y. Choquet-Bruhat, C. DeWitt-Morette, and M. Dillard-Bleick, Analysis, Manifolds, and Physics (North Holland, Amsterdam, 1977).

[9] J. W. York, personal notes on the hyperbolic formulation of general relativity (tensorindex construction), February 1995.

[10] S. R. Lau, Canonical Variables and Quasilocal Energy in General Relativity, University of North Carolina Ph.D. dissertation, June 1994.

[11] M. Göckeler and T. Schücker, Differential Geometry, Gauge Theories, and Gravity (Cambridge University Press, Cambridge, 1987).

[12] J. W. York in Sources of Gravitational Radiation, edited by L. L. Smarr (Cambridge University Press, Cambridge, 1979).

[13] J. Frauendiener and L. J. Mason, in Twistors in Mathematics and Physics, edited by T. N. Bailey and R. J. Bastion (Cambridge University Press, Cambridge, 1990).

[14] C. W. Misner, K. S. Thorne, and J. A. Wheeler, Gravitation (Freeman, San Francisco, 1973). 